\title{
The effects of beta-blocker use on cancer prognosis: a meta-analysis based on 3/9,006 patients
}

This article was published in the following Dove Press journal:

OncoTargets and Therapy

\section{Zhijing $\mathrm{Na},{ }^{1}, *$ Xinbo \\ Qiao, ${ }^{1, *}$ Xuanyu Hao, ${ }^{2}$ \\ Ling Fan, ${ }^{3}$ Yao Xiao, ${ }^{4}$ Yining \\ Shao, ${ }^{4}$ Mingwei Sun, ${ }^{4}$ Ziyi \\ Feng, ${ }^{4}$ Wen Guo, ${ }^{4}$ Jiapo Li, \\ Jiatong Li, ${ }^{5}$ Dongyang $\mathrm{Li}^{6}$ \\ 'Department of Post-graduate, Shengjing Hospital of China Medical University, Shenyang, Liaoning I I 0004, People's Republic of China; 2Department of Rheumatology and Immunology, Shengjing Hospital of China Medical University, Shenyang, Liaoning I 10022, People's Republic of China; ${ }^{3}$ Department of Nursing, Shengjing Hospital of China Medical University, Shenyang, Liaoning I 10004 , People's Republic of China; ${ }^{4}$ The First Clinical Department of China Medical University, Shenyang, Liaoning I I0I22, People's Republic of China; ${ }^{5}$ Department of Undergraduate, The First Clinical Academy of China Medical University, Shenyang, Liaoning I I000 I, People's Republic of China; ${ }^{6}$ Department of Urology, Shengjing Hospital of China Medical University, Shenyang, Liaoning I I0004, People's Republic of China}

*These authors contributed equally to this work

Correspondence: Dongyang Li Department of Urology, Shenging Hospital of China Medical University, No. 36 Sanhao Street, Heping District, Shenyang, Liaoning, I I0004, People's Republic of China

Tel/fax +86 24966153421 I

Email dyli@cmu.edu.cn
Background: Beta-blockers are antihypertensive drugs and have shown potential in cancer prognosis. However, this benefit has not been well defined due to inconsistent results from the published studies.

Methods: To investigate the association between administration of beta-blocker and cancer prognosis, we performed a meta-analysis. A literature search of PubMed, Embase, Cochrane Library, and Web of Science was conducted to identify all relevant studies published up to September 1, 2017. Thirty-six studies involving 319,006 patients were included. Hazard ratios were pooled using a random-effects model. Subgroup analyses were conducted by stratifying ethnicity, duration of drug use, cancer stage, sample size, beta-blocker type, chronological order of drug use, and different types of cancers.

Results: Overall, there was no evidence to suggest an association between beta-blocker use and overall survival ( $\mathrm{HR}=0.94,95 \% \mathrm{CI}$ : $0.87-1.03$ ), all-cause mortality $(\mathrm{HR}=0.99,95 \% \mathrm{CI}$ : 0.94-1.05), disease-free survival ( $\mathrm{HR}=0.59,95 \% \mathrm{CI}: 0.30-1.17)$, progression-free survival (HR $=0.90,95 \%$ CI: 0.79-1.02), and recurrence-free survival ( $\mathrm{HR}=0.99,95 \% \mathrm{CI}: 0.76-1.28)$, as well. In contrast, beta-blocker use was significantly associated with better cancer-specific survival (CSS) (HR=0.78, 95\% CI: 0.65-0.95). Subgroup analysis generally supported main results. But there is still heterogeneity among cancer types that beta-blocker use is associated with improved survival among patients with ovarian cancer, pancreatic cancer, and melanoma.

Conclusion: The present meta-analysis generally demonstrates no association between betablocker use and cancer prognosis except for CSS in all population groups examined. High-quality studies should be conducted to confirm this conclusion in future.

Keywords: cancer, prognosis, beta-blocker, meta-analysis

\section{Introduction}

Cancer is the main disease that endangers human life worldwide. The incidence of cancer remains grim that 1.7 million new cancer cases and 0.6 million cancer deaths are projected to occur in USA in 2017. ${ }^{1}$ Since cancer often leads to poor survival and a marked decline in quality of life, effective and safe therapies for prolonging cancer survival are urgently needed.

Beta-blockers have been considered as a safe cardiovascular treatment for decades. ${ }^{2}$ At present, the beta-adrenergic receptor downstream signaling pathway is certified as an important regulator of progression and metastasis of some important tumors, ${ }^{3}$ making beta-blockers a new alternative for cancer adjuvant chemotherapy. ${ }^{4}$ So far, a growing number of studies have supported the use of beta-blockers in prolonging survival of cancer patients, ${ }^{8-30}$ but several studies have put forward controversial conclusions. ${ }^{31-43}$ 
The purpose of this study was to use meta-analysis to quantitatively and comprehensively summarize the evidence for the relationship between beta-blocker exposure and survival outcomes of various cancers.

\section{Materials and methods}

\section{Search strategy}

Under the guidance of the Preferred Reporting Items for Systematic Reviews and Meta-Analyses (PRISMA), we conducted this meta-analysis. To identify the studies of interest, we systematically searched PubMed (Supplementary material online file), Embase, Cochrane Library, and Web of Science for research reports published up to September 1, 2017. Search terms included: Adrenergic beta-Antagonist(s), beta-blocker(s), atenolol, bisoprolol, carvedilol, metoprolol, propranolol, sotalol, timolol, arotinolol, betaxolol, bevantolol, carteolol or celiprolol\} combined with \{cancer(s), carcinoma(s), malignancy(ies), neoplasm(s) or tumour(s)\} and \{prognosis, survival or mortality . We scanned the titles and abstracts of the studies identified in the initial search, excluding those apparently unrelated. The full text of the remaining articles was read to determine the studies that can be included. In addition, we have further studied the reference lists of articles for additional studies.

\section{Inclusion and exclusion criteria}

Our inclusion criteria were: 1) case-control or cohort studies or randomized controlled trials (RCTs); 2) patients with cancer; 3) reported at least 20 patients; 4) evaluated the therapeutic value of beta-blockers in cancer prognosis; 5) compared beta-blocker users with non-users in patients; 6) reported survival outcomes like overall survival (OS), all-cause mortality, cancer-specific survival (CSS), diseasefree survival (DFS), progression-free survival (PFS), and recurrence-free survival (RFS); 7) reported HR with 95\% CI for survival of comparison between exposure group and control group or HR could be obtained from other sufficient information.

Articles were excluded from the analyses for any of the following reasons: 1) reviews, commentaries, experimental laboratory articles, animal studies, or letters; 2) repeated publications; 3) impossible to calculate HR with $95 \%$ CI for survival from the paper.

\section{Data extraction}

The following information was extracted from each study: 1) publication data: first author's name, publication year, and geographical location of the study; 2) study design; 3 ) number and characteristics of participants; 4) types of beta-blockers used; 5) HR estimates with their 95\% CIs and control for multiple factors by matching or adjustments. If the HR and 95\% CI could not be obtained directly, they were estimated from Kaplan-Meier curves. ${ }^{5}$

\section{Quality assessment}

Quality of the included studies was assessed using the Newcastle-Ottawa Quality Assessment Scale (NOS). Studies of medium quality scored 6-7 points. This assessment was completed by two investigators ( $\mathrm{ZN}$ and $\mathrm{XQ}$ ) independently, and any disagreements were solved by a revaluation of the original article with a third author $(\mathrm{XH})$.

\section{Statistical analysis}

For the meta-analysis, we calculated pooled HRs with $95 \%$ CI for all the studies. We used the Cochran's $Q$-test to examine whether the results of the studies were homogeneous. The $P$-value $<0.10$ for $Q$-test indicated heterogeneity. Quantity of $I^{2}$ was also calculated to describe the percentage variation across studies due to heterogeneity. We regarded an $I^{2}$ value $>50 \%$ as indicative of significant heterogeneity. A fixed-effects model (inverse variance method) was used to calculate pooled results when no heterogeneity existed among the included studies; otherwise, a randomeffects model (DerSimonian and Laird method) was used with the weights inversely proportional to the variance of hazard ratio of each trial. ${ }^{6,7}$ To identify potential sources of between-study heterogeneity, subgroup analyses were conducted by stratifying ethnicity, duration of drug use, cancer stage, sample size, beta-blocker type, chronological order of drug use, and different types of cancers. We conducted sensitivity analysis to determine the relative effect of a particular study on the meta-analysis model. To assess the influence of potential causes, meta-regression models were fitted separately for each cause except for beta-blocker therapy. The Begg's adjusted rank correlation test and the Egger's regression asymmetry tests were used to evaluate the effects of publication bias. All analyses were conducted using Stata 12.0 software (Markummitchell, Torrance, CA, USA), and we read Kaplan-Meier curves with Engauge Digitizer version 9.8 .

\section{Results}

\section{Study search and characteristics}

The flow of literature selection applying the systematic search and selection strategies to identify qualified reports 
is shown in Figure 1. Six hundred and thirty studies were initially identified by the search. Of these, we retrieved 49 potential studies by filtering the titles and abstracts. Due to insufficient information (12 studies) or including the same patients (one study), 13 studies were excluded after further comprehensive review. Two studies were conducted in the same institute, but as the sample patients were at different stages and were treated differently, we considered them to be different cohorts. ${ }^{89}$ Finally, a total of 36 studies were included in the pooled analyses.

Table 1 showed the characteristics of the 36 studies. The articles were published from 2011 to 2017, which included 319,006 patients. Of them, 35 studies utilized cohort design ${ }^{8}$ ${ }^{10,12-43}$ and one study used case-control design. ${ }^{11}$ Besides, there were 22 hospital-based studies ${ }^{9-11,14-16,18,19,21,23,24,26-31,33-}$ $35,40,41$ and 14 population-based studies. , $12,13,17,20,22,25,32,36-39,42,43$ Overall, all the 36 studies reported the prognostic value of beta-blockers in the survival of cancer patients.

\section{Quality assessment}

While there was small variation in the methodological quality of the included studies, all 36 included studies were judged as moderate to relative high quality according to the NOS assessment tool, with scores of 6 (11 studies), 7 (20 studies), and 8 (five studies, Table S1).

\section{Beta-blockers and survival of cancer Meta-analysis of overall survival}

As displayed in Figure 2A, the forest plot showed that betablocker use was not associated with OS. The pooled HR was 0.94 (95\% CI: 0.87-1.03, $P=0.172$ ) from 22 observational studies. Considering the high heterogeneity $\left(I^{2}=83.3 \%, P<0.001\right)$, we used random-effects model to pool the studies.

\section{Meta-analysis of all-cause mortality}

Twelve studies focused on beta-blocker use and allcause mortality. A random-effects model was used and

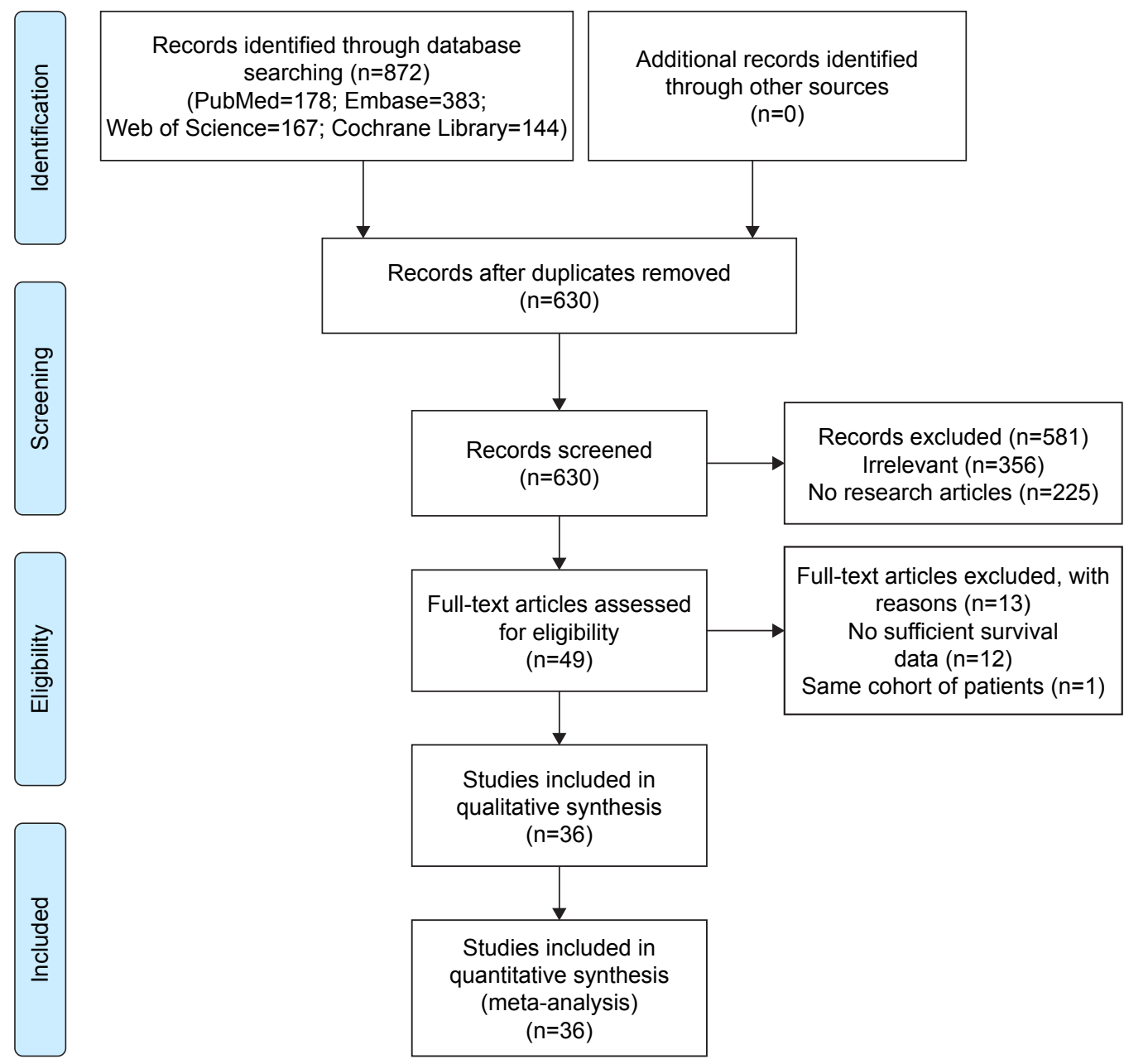

Figure I PRISMA flowchart of article selection for this meta-analysis.

Abbreviation: PRISMA, Preferred Reporting Items for Systematic Reviews and Meta-Analyses. 
Table I Characteristics of studies included for meta-analysis

\begin{tabular}{|c|c|c|c|c|c|c|c|c|c|c|}
\hline Reference & Study & Country & Duration & $\begin{array}{l}\text { Sample } \\
\text { size }\end{array}$ & $\begin{array}{l}\text { Median } \\
\text { age } \\
\text { (years) }\end{array}$ & $\begin{array}{l}\text { Study } \\
\text { design }\end{array}$ & Cancer type & Stage & Surgery & $\begin{array}{l}\text { Beta-blocker } \\
\text { type }\end{array}$ \\
\hline 8 & $\begin{array}{l}\text { Grytli et al } \\
(2013)\end{array}$ & Norway & 2004-2009 & 655 & 72 & PB cohort & Prostate cancer & $\begin{array}{l}\text { I/II 60.1\%, } \\
\text { III/IV 39.9\% }\end{array}$ & NR & $\begin{array}{l}\text { Mixed: beta } \\
\text { selective }(80.2 \%) ; \\
\text { non-selective } \\
(19.8 \%)\end{array}$ \\
\hline 9 & $\begin{array}{l}\text { Grytli et al } \\
(2014)\end{array}$ & Norway & $2000-2011$ & 3,561 & 76.3 & HB cohort & Prostate cancer & $\begin{array}{l}\leq \text { T2A } 14.9 \% \\
\text { T2b-T2c } 18.5 \% ; \\
\geq \text { T3a } 66.6 \%\end{array}$ & NR & $\begin{array}{l}\text { Mixed: beta I } \\
\text { selective (77.9\%); } \\
\text { non-selective } \\
\text { (3.0\%); alpha and } \\
\text { beta mixed (4.5\%) }\end{array}$ \\
\hline
\end{tabular}

\begin{tabular}{|c|c|c|c|c|c|c|c|c|}
\hline 10 & $\begin{array}{l}\text { Al-Niaimi et al USA } \\
(2016)\end{array}$ & $2000-2010 \quad 185$ & $\begin{array}{l}66.2 \\
63.8\end{array}$ & HB cohort & Ovarian cancer & $\begin{array}{l}\mathrm{I} / \mathrm{II} 26 \% \\
\text { III/IV } 74 \%\end{array}$ & Yes & NR \\
\hline II & $\begin{array}{ll}\text { Aydiner et al } & \text { Turkey } \\
(2013) & \end{array}$ & $2003-20 I I \quad I 07$ & $\begin{array}{l}61 \\
(42-8 I)\end{array}$ & $\begin{array}{l}\text { HB case- } \\
\text { control }\end{array}$ & $\begin{array}{l}\text { Non-small-cell } \\
\text { lung cancer }\end{array}$ & NR & Mixed & Mixed \\
\hline
\end{tabular}

\begin{tabular}{|c|c|c|c|c|c|c|c|c|c|}
\hline 12 & $\begin{array}{l}\text { Barron et al } \\
(2011)\end{array}$ & Ireland/USA & $200 \mathrm{I}-20064,808$ & 69.171 & PB cohort & Breast cancer & $\begin{array}{l}\text { I/II 75.6\%, } \\
\text { III/IV 24.4\% }\end{array}$ & NR & Beta non-selective \\
\hline 12 & $\begin{array}{l}\text { Barron et al } \\
(2011)\end{array}$ & Ireland/USA & $200 \mathrm{I}-20065,263$ & 69.171 & PB cohort & Breast cancer & $\begin{array}{l}\text { I/II 75.6\%, } \\
\text { III/IV 24.4\% }\end{array}$ & NR & Beta selective \\
\hline 12 & $\begin{array}{l}\text { Barron et al } \\
(2011)\end{array}$ & Ireland/USA & $200 \mathrm{I}-20065,80 \mathrm{I}$ & 69.171 & PB cohort & Breast cancer & $\begin{array}{l}\text { I/II 75.6\%, } \\
\text { III/IV 24.4\% }\end{array}$ & NR & $\begin{array}{l}\text { Mixed: beta } \\
\text { selective (88\%); } \\
\text { non-selective } \\
(12 \%)\end{array}$ \\
\hline 13 & $\begin{array}{l}\text { Beg et al } \\
(2017)\end{array}$ & USA & $2006-2009 \quad 13,702$ & 76 & PB cohort & $\begin{array}{l}\text { Pancreatic } \\
\text { adenocarcinoma }\end{array}$ & $\begin{array}{l}/ / I 138.1 \%, \\
\text { III/IV 6I.9\% }\end{array}$ & $\begin{array}{l}\text { Mixed } \\
69.3 \%\end{array}$ & NR \\
\hline 14 & $\begin{array}{l}\text { Bir et al } \\
(2015)\end{array}$ & USA & $200 I-20 I 3225$ & $\begin{array}{l}57.34 \pm \\
10.98\end{array}$ & HB cohort & $\begin{array}{l}\text { Metastatic brain } \\
\text { tumors }\end{array}$ & NR & Yes & Beta I selective \\
\hline 15 & $\begin{array}{l}\text { De Giorgi } \\
\text { et al }(20 / 3)\end{array}$ & Italy & $|993-200974|$ & 6453 & HB cohort & Thick melanoma & NR & Mixed & $\begin{array}{l}\text { Mixed: beta I } \\
\text { selective (73\%); } \\
\text { non-selective } \\
(27 \%)\end{array}$ \\
\hline 16 & $\begin{array}{l}\text { Diaz et al } \\
(20 \mid 2)\end{array}$ & USA & $1996-2006248$ & 67 & HB cohort & Ovarian cancer & III/IV I00\% & Yes & $\begin{array}{l}\text { Mixed: beta I } \\
\text { selective (75\%); } \\
\text { non-selective } \\
\text { (I3\%); mixed } \\
\text { alpha and beta } \\
\text { adrenergic } \\
\text { antagonist (I3\%) }\end{array}$ \\
\hline
\end{tabular}




\begin{tabular}{|c|c|c|c|c|c|c|c|c|c|c|}
\hline \multicolumn{2}{|c|}{ No. of patients } & \multirow{2}{*}{$\begin{array}{l}\text { Exposure } \\
\text { category }\end{array}$} & \multirow{2}{*}{$\begin{array}{l}\text { Follow- } \\
\text { up time } \\
\text { (months) }\end{array}$} & \multirow[t]{2}{*}{ Treatment } & \multirow[t]{2}{*}{ HR } & \multirow[t]{2}{*}{$95 \% \mathrm{Cl}$} & \multirow{2}{*}{$\begin{array}{l}\text { Survival } \\
\text { outcome }\end{array}$} & \multirow{2}{*}{$\begin{array}{l}\text { Multivariable } \\
\text { analysis }\end{array}$} & \multirow{2}{*}{ Adjusted for } & \multirow{2}{*}{$\begin{array}{l}\text { Study } \\
\text { quality } \\
\text { (NOS } \\
\text { score) }\end{array}$} \\
\hline Exposure & Control & & & & & & & & & \\
\hline \multirow[t]{2}{*}{80} & 575 & $\begin{array}{l}\text { Pre-diagnostic } \\
\text { beta-blocker } \\
\text { use }\end{array}$ & 122 & ADT or not & 0.88 & $\begin{array}{l}0.56- \\
1.38\end{array}$ & OS & Yes & $\begin{array}{l}\text { Age at diagnosis, metastasis } \\
\text { at diagnosis, and level of } \\
\text { education }\end{array}$ & 8 \\
\hline & & & & & 0.79 & $\begin{array}{l}0.68- \\
0.91\end{array}$ & CSS & & & \\
\hline \multirow[t]{2}{*}{1,115} & 2,446 & $\begin{array}{l}\text { Pre-diagnostic } \\
\text { beta-blocker } \\
\text { use }\end{array}$ & 39 & $\begin{array}{l}\text { RT or radical } \\
\text { prostatectomy }\end{array}$ & 0.96 & $\begin{array}{l}0.87- \\
1.05\end{array}$ & OS & Yes & $\begin{array}{l}\text { Age, prostate-specific } \\
\text { antigen level, Gleason score, } \\
\text { clinical T stage, presence } \\
\text { and type of metastases, } \\
\text { performance status, and } \\
\text { androgen deprivation, } \\
\text { therapy initiated within } \\
6 \text { months after diagnosis }\end{array}$ & 7 \\
\hline & & & & & 0.97 & $\begin{array}{l}0.72- \\
1.31\end{array}$ & CSS & & & \\
\hline 70 & 115 & $\begin{array}{l}\text { Post-diagnostic } \\
\text { beta-blocker } \\
\text { use (time- } \\
\text { dependent) }\end{array}$ & 91 & $\mathrm{CT}$ & 0.68 & $\begin{array}{l}0.46- \\
0.99\end{array}$ & OS & Yes & $\begin{array}{l}\text { Age, stage, grade, } \\
\text { cytoreduction status, BMI, } \\
\text { and presence or absence of } \\
\text { diabetes }\end{array}$ & 7 \\
\hline 35 & 72 & $\begin{array}{l}\text { Post-diagnostic } \\
\text { beta-blocker } \\
\text { use (time- } \\
\text { fixed) }\end{array}$ & $\begin{array}{l}17.8 \\
(1-102)\end{array}$ & $\mathrm{CT}$ & 0.69 & $\begin{array}{l}0.36- \\
1.34\end{array}$ & OS & Yes & $\begin{array}{l}\text { Age, sex, performance } \\
\text { status, histologic subtype, } \\
\text { smoking status, presence of } \\
\text { comorbidities (COPD, IHD, } \\
\text { HT, and DM) }\end{array}$ & 7 \\
\hline 70 & 4,738 & $\begin{array}{l}\text { Pre-diagnostic } \\
\text { beta-blocker } \\
\text { use }\end{array}$ & $\begin{array}{l}4243.2 \\
32.436\end{array}$ & CT or not & 0.19 & $\begin{array}{l}0.06- \\
0.60\end{array}$ & OS & Yes & $\begin{array}{l}\text { Age, stage, grade, and } \\
\text { comorbidity }\end{array}$ & 7 \\
\hline 525 & 4,738 & $\begin{array}{l}\text { Pre-diagnostic } \\
\text { beta-blocker } \\
\text { use }\end{array}$ & $\begin{array}{l}4243.2 \\
32.436\end{array}$ & CT or not & 1.08 & $\begin{array}{l}0.84- \\
1.40\end{array}$ & OS & Yes & $\begin{array}{l}\text { Age, stage, grade, and } \\
\text { comorbidity }\end{array}$ & 7 \\
\hline 595 & 4,738 & $\begin{array}{l}\text { Pre-diagnostic } \\
\text { beta-blocker } \\
\text { use }\end{array}$ & $\begin{array}{l}4243.2 \\
32.436\end{array}$ & CT or not & 1.08 & $\begin{array}{l}0.84 \\
1.39\end{array}$ & CSS & Yes & $\begin{array}{l}\text { Age, stage, grade, and } \\
\text { comorbidity }\end{array}$ & 7 \\
\hline 5,209 & 8,493 & NR & NR & NR & 0.9 & $\begin{array}{l}0.85- \\
0.95\end{array}$ & OS & Yes & $\begin{array}{l}\text { Age, sex, race, stage at } \\
\text { diagnosis, site of cancer, and } \\
\text { Charlson comorbidity index }\end{array}$ & 8 \\
\hline 40 & 185 & NR & 10.57 & GKRS & 1.08 & $\begin{array}{l}0.65- \\
1.79\end{array}$ & OS & Yes & $\begin{array}{l}\text { MBT kind, metastasis, tumor } \\
\text { recurrence, tumor response, } \\
\text { GKRS, prognostic factor }\end{array}$ & 7 \\
\hline 79 & 662 & $\begin{array}{l}\text { Post-diagnostic } \\
\text { beta-blocker } \\
\text { use (time- } \\
\text { dependent) }\end{array}$ & 50.4 & NR & 0.03 & $\begin{array}{l}0.01- \\
0.17\end{array}$ & DFS & Yes & $\begin{array}{l}\text { Age, Breslow thickness, and } \\
\text { ulceration }\end{array}$ & 7 \\
\hline & & & & & 0.04 & $\begin{array}{l}0.00- \\
0.38\end{array}$ & OS & & & \\
\hline 23 & 225 & NR & NR & $\mathrm{CT}$ & 0.56 & $\begin{array}{l}0.43- \\
1.26\end{array}$ & OS & Yes & $\begin{array}{l}\text { Age, stage, grade, and } \\
\text { cytoreduction status }\end{array}$ & 6 \\
\hline
\end{tabular}


Table I (Continued)

\begin{tabular}{|c|c|c|c|c|c|c|c|c|c|c|}
\hline Reference & Study & Country & Duration & $\begin{array}{l}\text { Sample } \\
\text { size }\end{array}$ & $\begin{array}{l}\text { Median } \\
\text { age } \\
\text { (years) }\end{array}$ & $\begin{array}{l}\text { Study } \\
\text { design }\end{array}$ & Cancer type & Stage & Surgery & $\begin{array}{l}\text { Beta-blocker } \\
\text { type }\end{array}$ \\
\hline 17 & $\begin{array}{l}\text { Ganz et al } \\
(2011)\end{array}$ & USA & 1997-2002 & 1,779 & NR & PB cohort & Breast cancer & $\begin{array}{l}\text { I/II } 96.9 \%, \\
\text { III/IV } 3.1 \%\end{array}$ & NR & $\begin{array}{l}\text { Mixed: beta } \\
\text { selective (86\%); } \\
\text { non-selective } \\
(14 \%)\end{array}$ \\
\hline
\end{tabular}

\begin{tabular}{llcccccc}
\hline 18 & $\begin{array}{l}\text { Giampieri et al Italy } \\
(2015)\end{array}$ & $2010-2013235$ & NR & HB cohort $\begin{array}{l}\text { Colorectal } \\
\text { cancer }\end{array}$ & NR & NR & NR
\end{tabular}

Tha

\begin{tabular}{|c|c|c|c|c|c|c|c|}
\hline 19 & $\begin{array}{l}\text { Hwa et al } \\
(2017)\end{array}$ & USA & $|995-2010 \quad 1,97|$ & 64 & HB cohort Myeloma & $\begin{array}{l}\text { I/II 75\%, } \\
\text { III/IV 25\% }\end{array}$ & Mixed \\
\hline
\end{tabular}

\begin{tabular}{|c|c|c|c|c|c|c|c|c|c|}
\hline 20 & $\begin{array}{l}\text { Jansen et al } \\
(2014)\end{array}$ & Germany & 2003-2007 I,975 & 68 & PB cohort & $\begin{array}{l}\text { Colorectal } \\
\text { cancer }\end{array}$ & $\begin{array}{l}\text { I/II 55\% } \\
\text { III/IV 45\% }\end{array}$ & $\begin{array}{l}\text { Mixed } \\
97.3 \%\end{array}$ & $\begin{array}{l}\text { Mixed: beta } \\
\text { selective (86\%); } \\
\text { non-selective } \\
(14 \%)\end{array}$ \\
\hline
\end{tabular}

(14\%)

\begin{tabular}{|c|c|c|c|c|c|c|c|c|c|}
\hline 21 & $\begin{array}{l}\text { Kim et al } \\
(20 \mid 7)\end{array}$ & Korea & $200 \mathrm{I}-20 \mathrm{I} 2 \mathrm{I}, 274$ & $\begin{array}{l}61 \\
(20-87)\end{array}$ & HB cohort & $\begin{array}{l}\text { Head and neck } \\
\text { squamous cell } \\
\text { carcinoma } \\
\text { (HNSCC) }\end{array}$ & $\begin{array}{l}\text { I/II 4I.4\% } \\
\text { III/IV 58.6\% }\end{array}$ & $\begin{array}{l}\text { Mixed } \\
69.2 \%\end{array}$ & $\begin{array}{l}\text { Mixed: beta I } \\
\text { selective (84\%); } \\
\text { non-selective } \\
(16 \%)\end{array}$ \\
\hline
\end{tabular}




\begin{tabular}{|c|c|c|c|c|c|c|c|c|c|c|}
\hline \multicolumn{2}{|c|}{ No. of patients } & \multirow{2}{*}{$\begin{array}{l}\text { Exposure } \\
\text { category }\end{array}$} & \multirow{2}{*}{$\begin{array}{l}\text { Follow- } \\
\text { up time } \\
\text { (months) }\end{array}$} & \multirow[t]{2}{*}{ Treatment } & \multirow[t]{2}{*}{ HR } & \multirow[t]{2}{*}{$95 \% \mathrm{Cl}$} & \multirow{2}{*}{$\begin{array}{l}\text { Survival } \\
\text { outcome }\end{array}$} & \multirow{2}{*}{$\begin{array}{l}\text { Multivariable } \\
\text { analysis }\end{array}$} & \multirow{2}{*}{ Adjusted for } & \multirow{2}{*}{$\begin{array}{l}\text { Study } \\
\text { quality } \\
\text { (NOS } \\
\text { score) }\end{array}$} \\
\hline Exposure & Control & & & & & & & & & \\
\hline \multirow[t]{3}{*}{204} & I,372 & NR & 98.4 & $\begin{array}{l}\mathrm{CT}, \mathrm{RT} \text {, both or } \\
\text { none }\end{array}$ & 1.04 & $\begin{array}{l}0.72- \\
1.5 \mathrm{|}\end{array}$ & OS & Yes & $\begin{array}{l}\text { Age at diagnosis, race, stage } \\
\text { of disease, pre-diagnosis } \\
\text { BMI, adjuvant treatment, } \\
\text { hormone receptor status, } \\
\text { tamoxifen use, and self- } \\
\text { reported hypertension and } \\
\text { diabetes }\end{array}$ & 8 \\
\hline & & & & & 0.86 & $\begin{array}{l}0.57- \\
1.32\end{array}$ & RFS & & & \\
\hline & & & & & 0.76 & $\begin{array}{l}0.44- \\
1.33\end{array}$ & CSS & & & \\
\hline \multirow[t]{2}{*}{29} & 206 & $\begin{array}{l}\text { Pre-diagnostic } \\
\text { beta-blocker } \\
\text { use }\end{array}$ & NR & $\begin{array}{l}\mathrm{CT} \text { or with } \\
\text { bevacizumab }\end{array}$ & $1.5 \mathrm{I}$ & $\begin{array}{l}0.88- \\
2.31\end{array}$ & OS & Yes & $\begin{array}{l}\text { Age, sex, and site of } \\
\text { metastases, previous } \\
\text { adjuvant chemotherapy, and } \\
\text { ECOG performance status }\end{array}$ & 7 \\
\hline & & & & & 1.19 & $\begin{array}{l}0.81- \\
1.72\end{array}$ & PFS & & & \\
\hline \multirow[t]{2}{*}{549} & 1,733 & $\begin{array}{l}\text { Post-diagnostic } \\
\text { beta-blocker } \\
\text { use (time- } \\
\text { fixed) }\end{array}$ & 74.3 & $\mathrm{CT}$ & 0.67 & $\begin{array}{l}0.55- \\
0.81\end{array}$ & OS & Yes & $\begin{array}{l}\text { Demographics, disease } \\
\text { characteristics, diagnosis } \\
\text { year, and various } \\
\text { chemotherapies }\end{array}$ & 7 \\
\hline & & & & & 0.53 & $\begin{array}{l}0.42- \\
0.67\end{array}$ & CSS & & & \\
\hline \multirow[t]{2}{*}{509} & $I, 3 I I$ & $\begin{array}{l}\text { Pre-diagnostic } \\
\text { beta-blocker } \\
\text { use }\end{array}$ & 60 & $\mathrm{CT}$ or RT & 0.99 & $\begin{array}{l}0.79- \\
1.22\end{array}$ & OS & Yes & $\begin{array}{l}\text { Age at diagnosis, sex, Union } \\
\text { for International Cancer } \\
\text { Control (UICC) stage (I-IV), } \\
\text { surgery, chemotherapy, } \\
\text { radiotherapy, body mass } \\
\text { index, hypertension, CVD } \\
\text { (including heart failure, } \\
\text { myocardial infarction, stroke, } \\
\text { and cardiac circulatory } \\
\text { disorder), diabetes, regular } \\
\text { use of nonsteroidal anti- } \\
\text { inflammatory drugs } \\
\text { (NSAIDs) including aspirin, } \\
\text { regular use of statins, use } \\
\text { of hormone replacement } \\
\text { therapy (HRT), lifetime } \\
\text { pack-years of active smoking, } \\
\text { physical activity (quartiles } \\
\text { of lifetime metabolic } \\
\text { equivalents [METs] in hours } \\
\text { per week), and participation } \\
\text { in health check-up }\end{array}$ & 8 \\
\hline & & & & & 0.93 & $\begin{array}{l}0.7 \mathrm{I}- \\
1.21\end{array}$ & CSS & & & \\
\hline 114 & $\mathrm{I}, \mathrm{I} 60$ & $\begin{array}{l}\text { Post-diagnostic } \\
\text { beta-blocker } \\
\text { use (time- } \\
\text { fixed) }\end{array}$ & 98 & $\begin{array}{l}\text { Primary curative } \\
\text { surgery, RT, } \\
\text { CRT with or } \\
\text { without IC, or } \\
\text { a combination } \\
\text { of these } \\
\text { treatments }\end{array}$ & 1.33 & $\begin{array}{l}0.93- \\
1.91\end{array}$ & DFS & Yes & $\begin{array}{l}\text { Age, sex, BMI, CCl, smoking, } \\
\text { alcohol, tumor site, tumor } \\
\text { classification T3-4, nodal } \\
\text { classification NI-3, overall } \\
\text { TNM stage III-IV, primary } \\
\text { treatment, second primary } \\
\text { cancer, hypertension }\end{array}$ & 6 \\
\hline
\end{tabular}


Table I (Continued)

\begin{tabular}{lccccc}
\hline Reference Study & Country & $\begin{array}{c}\text { Duration Sample Median Study } \\
\text { size } \begin{array}{l}\text { age } \\
\text { (years) }\end{array}\end{array}$ & Cancer type Stage & $\begin{array}{l}\text { Surgery Beta-blocker } \\
\text { type }\end{array}$
\end{tabular}

\begin{tabular}{|c|c|c|c|c|c|c|c|c|}
\hline 22 & $\begin{array}{l}\text { Lemeshow } \\
\text { et al (20II) }\end{array}$ & Denmark & Since 19434,179 & 66 & PB cohort & Melanoma & $\begin{array}{l}\text { I/II 63.8\%, } \\
\text { III/IV 36.2\% }\end{array}$ & Mixed \\
\hline
\end{tabular}

\begin{tabular}{llllllll}
\hline 23 & Melhem- USA & I $995-2007$ I,4I3 & 5749 & HB cohort Breast cancer & I/II 55.6\%, & Yes & Mixed: beta \\
Bertrandt et al & & & & III/IV 44.4\% & selective (89\%); \\
$(201 \mathrm{I})$ & & & & non-selective
\end{tabular}

(II\%)

\begin{tabular}{|c|c|c|c|c|c|c|c|c|c|c|}
\hline 24 & $\begin{array}{l}\text { Springate et al } \\
\text { (2015) }\end{array}$ & NR & 1997-2006 & 11,302 & NR & HB cohort & Mixed cancer & NR & NR & Mixed \\
\hline 24 & $\begin{array}{l}\text { Springate et al } \\
(2015)\end{array}$ & NR & 1997-2006 & 6,274 & NR & HB cohort & Mixed cancer & NR & NR & Mixed \\
\hline 25 & $\begin{array}{l}\text { Udumyan et al } \\
(2017)\end{array}$ & Swedish & 2006-2009 & 2,394 & $\begin{array}{l}70.9 \\
67.1\end{array}$ & PB cohort & $\begin{array}{l}\text { Pancreatic } \\
\text { adenocarcinoma }\end{array}$ & $\begin{array}{l}\text { I/II 2I\%, } \\
\text { III/IV 79\% }\end{array}$ & NR & $\begin{array}{l}\text { Mixed: beta I } \\
\text { selective (89\%); } \\
\text { non-selective } \\
(11 \%)\end{array}$ \\
\hline 26 & $\begin{array}{l}\text { Wang et al } \\
(20 \mid 3)\end{array}$ & USA & $1998-2010$ & 722 & $\begin{array}{l}65 \\
(34-95)\end{array}$ & HB cohort & $\begin{array}{l}\text { Non-small-cell } \\
\text { lung cancer }\end{array}$ & $\begin{array}{l}\text { I/II 6.2\%, } \\
\text { III 93.8\% }\end{array}$ & Mixed & $\begin{array}{l}\text { Mixed: beta } \\
\text { selective (86\%); } \\
\text { non-selective } \\
(14 \%)\end{array}$ \\
\hline
\end{tabular}

\begin{tabular}{|c|c|c|c|c|c|c|c|c|c|}
\hline 27 & $\begin{array}{l}\text { Watkins et al } \\
(2015)\end{array}$ & USA & $2000-2010 \quad \mathrm{I}, 425$ & 61.668 & HB cohort & Ovarian cancer & $\begin{array}{l}\text { I/II I0\%, } \\
\text { III/IV 90\% }\end{array}$ & Yes & $\begin{array}{l}\text { Mixed: beta } \\
\text { selective (72. } 1 \%) ; \\
\text { non-selective } \\
(27.9 \%)\end{array}$ \\
\hline
\end{tabular}




\begin{tabular}{|c|c|c|c|c|c|c|c|c|c|c|}
\hline \multicolumn{2}{|c|}{ No. of patients } & \multirow{2}{*}{$\begin{array}{l}\text { Exposure } \\
\text { category }\end{array}$} & \multirow{2}{*}{$\begin{array}{l}\text { Follow- } \\
\text { up time } \\
\text { (months) }\end{array}$} & \multirow[t]{2}{*}{ Treatment } & \multirow[t]{2}{*}{ HR } & \multirow[t]{2}{*}{$95 \% \mathrm{Cl}$} & \multirow{2}{*}{$\begin{array}{l}\text { Survival } \\
\text { outcome }\end{array}$} & \multirow{2}{*}{$\begin{array}{l}\text { Multivariable } \\
\text { analysis }\end{array}$} & \multirow{2}{*}{ Adjusted for } & \multirow{2}{*}{$\begin{array}{l}\text { Study } \\
\text { quality } \\
\text { (NOS } \\
\text { score) }\end{array}$} \\
\hline Exposure & Control & & & & & & & & & \\
\hline & & & & & 1.49 & $\begin{array}{l}0.99- \\
2.22\end{array}$ & CSS & & & \\
\hline & & & & & 1.54 & $\begin{array}{l}1.17- \\
2.05\end{array}$ & OS & & & \\
\hline \multirow[t]{2}{*}{372} & 3,807 & $\begin{array}{l}\text { Pre-diagnostic } \\
\text { beta-blocker } \\
\text { use }\end{array}$ & 58.8 & NR & 0.81 & $\begin{array}{l}0.67- \\
0.97\end{array}$ & OS & Yes & $\begin{array}{l}\text { Age and comorbidity index } \\
\text { score }\end{array}$ & 7 \\
\hline & & & & & 0.87 & $\begin{array}{l}0.64- \\
1.2\end{array}$ & CSS & & & \\
\hline \multirow[t]{3}{*}{102} & $I, 31 I$ & $\begin{array}{l}\text { Post-diagnostic } \\
\text { beta-blocker } \\
\text { use (time- } \\
\text { fixed) }\end{array}$ & 58.8 & $\begin{array}{l}\text { Anthracylines } \\
\text { and taxane- } \\
\text { based } \\
\text { neoadjuvant CT }\end{array}$ & 0.3 & $\begin{array}{l}0.10 \\
0.87\end{array}$ & RFS & Yes & $\begin{array}{l}\text { Age, race, stage, } \\
\text { grade, receptor status, } \\
\text { lymphovascular invasion, } \\
\text { body mass index, diabetes, } \\
\text { hypertension, and } \\
\text { angiotensin-converting } \\
\text { enzyme inhibitor use }\end{array}$ & 7 \\
\hline & & & & & 0.76 & $\begin{array}{l}0.44- \\
1.33\end{array}$ & CSS & & & \\
\hline & & & & & 0.35 & $\begin{array}{l}0.12- \\
1.00\end{array}$ & OS & & & \\
\hline 4,030 & 7,272 & $\begin{array}{l}\text { Pre-diagnostic } \\
\text { beta-blocker } \\
\text { use }\end{array}$ & 2930 & NR & 1.03 & $\begin{array}{l}0.93- \\
1.14\end{array}$ & OS & No & No & 7 \\
\hline 1,406 & 4,868 & $\begin{array}{l}\text { Pre-diagnostic } \\
\text { beta-blocker } \\
\text { use }\end{array}$ & 2930 & NR & 1.18 & $\begin{array}{l}\mathrm{I} .04 \\
\mathrm{I} .33\end{array}$ & OS & No & No & 7 \\
\hline \multirow[t]{2}{*}{522} & $\mathrm{I}, 872$ & $\begin{array}{l}\text { Pre-diagnostic } \\
\text { beta-blocker } \\
\text { use }\end{array}$ & 5 & NR & 0.79 & $\begin{array}{l}0.70 \\
0.90\end{array}$ & OS & Yes & $\begin{array}{l}\text { Sociodemographic factors, } \\
\text { tumor characteristics, } \\
\text { comorbidity score, and } \\
\text { other medications }\end{array}$ & 8 \\
\hline & & & & & 0.77 & $\begin{array}{l}0.69- \\
0.87\end{array}$ & CSS & & & \\
\hline \multirow[t]{4}{*}{155} & 567 & $\begin{array}{l}\text { Post-diagnostic } \\
\text { beta-blocker } \\
\text { use (time- } \\
\text { fixed) }\end{array}$ & $44(I-155)$ & Definitive RT & 0.91 & $\begin{array}{l}0.64- \\
1.31\end{array}$ & PFS & Yes & $\begin{array}{l}\text { Age, Karnofsky performance } \\
\text { score, clinical stage, tumor } \\
\text { histology, use of concurrent } \\
\text { chemotherapy, radiation } \\
\text { dose, GTV, hypertension, } \\
\text { chronic obstructive } \\
\text { pulmonary disease, and } \\
\text { aspirin consumption }\end{array}$ & 7 \\
\hline & & & & & 0.67 & $\begin{array}{l}0.50- \\
0.91\end{array}$ & DMFS & & & \\
\hline & & & & & 0.74 & $\begin{array}{l}0.58- \\
0.95\end{array}$ & DFS & & & \\
\hline & & & & & 0.78 & $\begin{array}{l}0.63- \\
0.97 \\
\end{array}$ & OS & & & \\
\hline \multirow[t]{2}{*}{269} & $I, 156$ & $\begin{array}{l}\text { Post-diagnostic } \\
\text { beta-blocker } \\
\text { use (time- } \\
\text { fixed) }\end{array}$ & NR & $\mathrm{CT}$ & 0.26 & $\begin{array}{l}0.19- \\
0.37\end{array}$ & OS & No & No & 6 \\
\hline & & & & & 0.24 & $\begin{array}{l}0.17- \\
0.34\end{array}$ & CSS & & & \\
\hline
\end{tabular}


Table I (Continued)

\begin{tabular}{|c|c|c|c|c|c|c|c|c|c|c|}
\hline Reference & Study & Country & Duration & $\begin{array}{l}\text { Sample } \\
\text { size }\end{array}$ & $\begin{array}{l}\text { Median } \\
\text { age } \\
\text { (years) }\end{array}$ & $\begin{array}{l}\text { Study } \\
\text { design }\end{array}$ & Cancer type & Stage & Surgery & $\begin{array}{l}\text { Beta-blocker } \\
\text { type }\end{array}$ \\
\hline 28 & $\begin{array}{l}\text { Yusuf et al } \\
(2012)\end{array}$ & USA & $2000-2006$ & 456 & 67 & HB cohort & Mixed cancer & $N R$ & $N R$ & $N R$ \\
\hline 29 & $\begin{array}{l}\text { Botteri et al } \\
(2013)\end{array}$ & Italy & $1997-2006$ & 800 & 6259 & HB cohort & Breast cancer & $\begin{array}{l}\text { I/II 86\%, } \\
\text { III/IV I4\% }\end{array}$ & Yes & $\begin{array}{l}\text { Mixed: beta I } \\
\text { selective (84.1\%); } \\
\text { non-selective } \\
\text { (4\%); alpha and } \\
\text { beta mixed } \\
(11.9 \%)\end{array}$ \\
\hline 30 & $\begin{array}{l}\text { Spera et al } \\
(2017)\end{array}$ & Canada & NR & $\mathrm{I}, \mathrm{I} 44$ & 6053 & HB cohort & Breast cancer & NR & Yes & Mixed \\
\hline 31 & $\begin{array}{l}\text { Johannesdottir } \\
\text { et al (20I3) }\end{array}$ & Denmark & $1999-2010$ & 6,253 & 65 & HB cohort & Ovarian cancer & NR & Mixed & NR \\
\hline 31 & $\begin{array}{l}\text { Johannesdottir } \\
\text { et al }(20 \text { I3) }\end{array}$ & Denmark & $1999-2010$ & 6,539 & 65 & HB cohort & Ovarian cancer & NR & Mixed & NR \\
\hline 32 & $\begin{array}{l}\text { Assayag et al } \\
(2014)\end{array}$ & Canada/UK & $1998-2012$ & 6,270 & 72.3 & PB cohort & Prostate cancer & NR & Yes & $\begin{array}{l}\text { Mixed: beta } \\
\text { selective (59.4\%); } \\
\text { non-selective } \\
(40.6 \%)\end{array}$ \\
\hline
\end{tabular}

\begin{tabular}{|c|c|c|c|c|c|c|c|c|c|c|}
\hline 33 & $\begin{array}{l}\text { Cata et al } \\
(20 \mid 4)\end{array}$ & USA & NR & 391 & NR & HB cohort & $\begin{array}{l}\text { Non-small-cell } \\
\text { lung cancer }\end{array}$ & $\begin{array}{l}\text { I/II 75.2\%, } \\
\text { III } 24.8 \%\end{array}$ & Yes & Beta I selective \\
\hline
\end{tabular}

\begin{tabular}{llllllllll}
\hline 33 & $\begin{array}{l}\text { Cata et al } \\
(2014)\end{array}$ & USA & NR & 286 & NR & HB cohort $\begin{array}{l}\text { Non-small-cell } \\
\text { lung cancer }\end{array}$ & $\begin{array}{l}\text { IIII } 75.2 \%, \\
\text { II.8\% }\end{array}$ & Yes & Beta non-selective
\end{tabular}

\begin{tabular}{|c|c|c|c|c|c|c|c|c|c|c|}
\hline 34 & $\begin{array}{l}\text { Heitz et al } \\
(20 \mid 3)\end{array}$ & $\begin{array}{l}\text { Germany/ } \\
\text { Canada }\end{array}$ & NR & 381 & 60 & HB cohort & Ovarian cancer & $\begin{array}{l}/ I I \text { 6.5\%, } \\
\text { III/IV 93.5\% }\end{array}$ & Yes & $\begin{array}{l}\text { Mixed: beta } \\
\text { selective (84\%); } \\
\text { non-selective } \\
(16 \%)\end{array}$ \\
\hline
\end{tabular}




\begin{tabular}{|c|c|c|c|c|c|c|c|c|c|c|}
\hline \multicolumn{2}{|c|}{ No. of patients } & \multirow{2}{*}{$\begin{array}{l}\text { Exposure } \\
\text { category }\end{array}$} & \multirow{2}{*}{$\begin{array}{l}\text { Follow- } \\
\text { up time } \\
\text { (months) }\end{array}$} & \multirow{2}{*}{ Treatment } & \multirow[t]{2}{*}{ HR } & \multirow[t]{2}{*}{$95 \% \mathrm{Cl}$} & \multirow{2}{*}{$\begin{array}{l}\text { Survival } \\
\text { outcome }\end{array}$} & \multirow{2}{*}{$\begin{array}{l}\text { Multivariable } \\
\text { analysis }\end{array}$} & \multirow{2}{*}{ Adjusted for } & \multirow{2}{*}{$\begin{array}{l}\text { Study } \\
\text { quality } \\
\text { (NOS } \\
\text { score) }\end{array}$} \\
\hline Exposure & Control & & & & & & & & & \\
\hline 211 & 245 & NR & 1.2 & Chest RT or CT & 0.64 & $\begin{array}{l}0.51- \\
0.81\end{array}$ & OS & Yes & $\begin{array}{l}\text { Age, cancer status, } \\
\text { cancer type, previous } \\
\text { chemotherapy, chest } \\
\text { radiotherapy, hyperlipidemia }\end{array}$ & 6 \\
\hline 74 & 726 & $\begin{array}{l}\text { Pre-diagnostic } \\
\text { beta-blocker } \\
\text { use }\end{array}$ & 7267.2 & $\begin{array}{l}\text { Adjuvant CT } \\
\text { and RT }\end{array}$ & 0.42 & $\begin{array}{l}0.18 \\
0.97\end{array}$ & CSS & Yes & $\begin{array}{l}\text { Age, tumor stage, and } \\
\text { treatment, peritumoral } \\
\text { vascular invasion and use of } \\
\text { other antihypertensive drugs, } \\
\text { antithrombotics, and statins }\end{array}$ & 7 \\
\hline \multirow[t]{2}{*}{153} & 991 & $\begin{array}{l}\text { Pre/post- } \\
\text { diagnostic } \\
\text { beta-blocker } \\
\text { use (time- } \\
\text { dependent) }\end{array}$ & 25.1 & $\mathrm{CT}$ & 0.81 & $\begin{array}{l}0.66- \\
0.99\end{array}$ & PFS & Yes & $\begin{array}{l}\text { Treatment arm (RAM } \\
\text { vs } \mathrm{PBO}), \mathrm{HHRR} \text { status, } \\
\text { geographic region, THE }\end{array}$ & 7 \\
\hline & & & & & 1.05 & $\begin{array}{l}0.85- \\
1.29\end{array}$ & OS & & & \\
\hline 87 & 6,166 & $\begin{array}{l}\text { Pre-diagnostic } \\
\text { beta-blocker } \\
\text { use }\end{array}$ & 30.6 & HRT & 1.18 & $\begin{array}{l}0.90- \\
1.55\end{array}$ & OS & Yes & $\begin{array}{l}\text { Age, comorbidity level, prior } \\
\text { use of diuretics, year of } \\
\text { diagnosis, aspirin, and statins }\end{array}$ & 7 \\
\hline 373 & 6,166 & $\begin{array}{l}\text { Pre-diagnostic } \\
\text { beta-blocker } \\
\text { use }\end{array}$ & 30.6 & HRT & 1.17 & $\begin{array}{l}1.02- \\
1.34\end{array}$ & OS & Yes & $\begin{array}{l}\text { Age, comorbidity level, prior } \\
\text { use of diuretics, year of } \\
\text { diagnosis, aspirin, and statins }\end{array}$ & 7 \\
\hline \multirow[t]{2}{*}{673} & 1,088 & $\begin{array}{l}\text { Post-diagnostic } \\
\text { beta-blocker } \\
\text { use (time- } \\
\text { dependent) }\end{array}$ & 45.6 & $\begin{array}{l}\text { Prostatectomy, } \\
\text { RT, ADT, and } \\
\text { CT }\end{array}$ & 0.97 & $\begin{array}{l}0.8- \\
1.16\end{array}$ & OS & No & No & 7 \\
\hline & & & & & 0.97 & $\begin{array}{l}0.72- \\
1.31\end{array}$ & CSS & & & \\
\hline \multirow[t]{2}{*}{149} & 242 & NR & NR & NR & 1.304 & $\begin{array}{l}0.973- \\
1.747\end{array}$ & RFS & Yes & $\begin{array}{l}\text { Age, stage of disease, BMI, } \\
\text { ASA physical status, smoking } \\
\text { status, CAD, postoperative } \\
\text { radiation treatment, type of } \\
\text { surgery, and perioperative } \\
\text { blood transfusions }\end{array}$ & 7 \\
\hline & & & & & 1.335 & $\begin{array}{l}0.966- \\
1.846\end{array}$ & OS & & & \\
\hline \multirow[t]{2}{*}{44} & 242 & NR & NR & NR & 0.989 & $\begin{array}{l}0.639- \\
1.532\end{array}$ & RFS & Yes & $\begin{array}{l}\text { Age, stage of disease, BMI, } \\
\text { ASA physical status, smoking } \\
\text { status, CAD, postoperative } \\
\text { radiation treatment, type of } \\
\text { surgery, and perioperative } \\
\text { blood transfusions }\end{array}$ & 7 \\
\hline & & & & & $\mathrm{I} .108$ & $\begin{array}{l}0.678- \\
1.812 \\
\end{array}$ & OS & & & \\
\hline \multirow[t]{2}{*}{38} & 343 & $\begin{array}{l}\text { Post-diagnostic } \\
\text { beta-blocker } \\
\text { use (time- } \\
\text { fixed) }\end{array}$ & 17 & CT & 0.92 & $\begin{array}{l}0.65- \\
1.31\end{array}$ & PFS & Yes & $\begin{array}{l}\text { Age, stage, grade, and } \\
\text { cytoreduction status }\end{array}$ & 7 \\
\hline & & & & & 0.74 & $\begin{array}{l}0.49- \\
1.11\end{array}$ & OS & & & \\
\hline
\end{tabular}


Table I (Continued)

\begin{tabular}{|c|c|c|c|c|c|c|c|c|c|c|}
\hline Reference & Study & Country & Duration & $\begin{array}{l}\text { Sample } \\
\text { size }\end{array}$ & $\begin{array}{l}\text { Median } \\
\text { age } \\
\text { (years) }\end{array}$ & $\begin{array}{l}\text { Study } \\
\text { design }\end{array}$ & Cancer type & Stage & Surgery & $\begin{array}{l}\text { Beta-blocker } \\
\text { type }\end{array}$ \\
\hline 35 & $\begin{array}{l}\text { Heitz et al } \\
(2017)\end{array}$ & Germany & $1999-2014$ & 801 & $\begin{array}{l}58 \\
(19-90)\end{array}$ & HB cohort & Ovarian cancer & $\begin{array}{l}\text { I/II 43.3\%, } \\
\text { III/IV 56.7\% }\end{array}$ & Yes & Beta I selective \\
\hline
\end{tabular}

\begin{tabular}{|c|c|c|c|c|c|c|c|c|c|c|}
\hline 36 & $\begin{array}{l}\text { Holmes et al } \\
(2013)\end{array}$ & Canada & 2004-2008 & 2,433 & 68.3 & PB cohort & Breast cancer & NR & NR & Mixed \\
\hline 36 & $\begin{array}{l}\text { Holmes et al } \\
(2013)\end{array}$ & Canada & 2004-2008 & 2,016 & 68.3 & PB cohort & $\begin{array}{l}\text { Colorectal } \\
\text { cancer }\end{array}$ & NR & NR & Mixed \\
\hline 36 & $\begin{array}{l}\text { Holmes et al } \\
(2013)\end{array}$ & Canada & 2004-2008 & 2,125 & 68.3 & PB cohort & Lung cancer & NR & NR & Mixed \\
\hline 36 & $\begin{array}{l}\text { Holmes et al } \\
(20 \mid 3)\end{array}$ & Canada & 2004-2008 & $\mathrm{I}, 868$ & 68.3 & PB cohort & Prostate cancer & NR & NR & Mixed \\
\hline 37 & $\begin{array}{l}\text { Jansen et al } \\
(2017)\end{array}$ & $\begin{array}{l}\text { The } \\
\text { Netherlands }\end{array}$ & $|998-20| \mid$ & 2,530 & 7368 & PB cohort & $\begin{array}{l}\text { Colorectal } \\
\text { cancer }\end{array}$ & $\begin{array}{l}\text { I/II 55.7\%, } \\
\text { III/IV 44.3\% }\end{array}$ & $\begin{array}{l}\text { Mixed } \\
89.8 \%\end{array}$ & $\begin{array}{l}\text { Mixed: beta } \\
\text { selective ( } 55 \%) ; \\
\text { non-selective }\end{array}$ \\
\hline
\end{tabular}

\begin{tabular}{|c|c|c|c|c|c|c|c|c|c|}
\hline 37 & Jansen (20I7) & $\begin{array}{l}\text { The } \\
\text { Netherlands }\end{array}$ & $|998-20||\quad|, 374$ & 7368 & PB cohort & $\begin{array}{l}\text { Colorectal } \\
\text { cancer }\end{array}$ & $\begin{array}{l}\text { I/II 55.7\%, } \\
\text { III/IV 44.3\% }\end{array}$ & $\begin{array}{l}\text { Mixed } \\
89.8 \%\end{array}$ & $\begin{array}{l}\text { Mixed: beta } \\
\text { selective }(66 \%) ; \\
\text { non-selective } \\
(34 \%)\end{array}$ \\
\hline
\end{tabular}

(34\%) 


\begin{tabular}{|c|c|c|c|c|c|c|c|c|c|c|}
\hline \multicolumn{2}{|c|}{ No. of patients } & \multirow{2}{*}{$\begin{array}{l}\text { Exposure } \\
\text { category }\end{array}$} & \multirow{2}{*}{$\begin{array}{l}\text { Follow- } \\
\text { up time } \\
\text { (months) }\end{array}$} & \multirow[t]{2}{*}{ Treatment } & \multirow[t]{2}{*}{ HR } & \multirow[t]{2}{*}{$95 \% \mathrm{Cl}$} & \multirow{2}{*}{$\begin{array}{l}\text { Survival } \\
\text { outcome }\end{array}$} & \multirow{2}{*}{$\begin{array}{l}\text { Multivariable } \\
\text { analysis }\end{array}$} & \multirow{2}{*}{ Adjusted for } & \multirow{2}{*}{$\begin{array}{l}\text { Study } \\
\text { quality } \\
\text { (NOS } \\
\text { score) }\end{array}$} \\
\hline Exposure & Control & & & & & & & & & \\
\hline \multirow[t]{2}{*}{141} & 660 & NR & 40 & $\mathrm{CT}$ & 0.94 & $\begin{array}{l}0.69- \\
1.29\end{array}$ & OS & Yes & $\begin{array}{l}\text { Age, ECOG, ASA, Charlton } \\
\text { comorbidity score (metric), } \\
\text { tumor residuals, histology, } \\
\text { body mass index, and FIGO } \\
\text { stage }\end{array}$ & 7 \\
\hline & & & & & 0.95 & $\begin{array}{l}0.72- \\
1.27\end{array}$ & PFS & & & \\
\hline 123 & 2,310 & $\begin{array}{l}\text { Pre-diagnostic } \\
\text { beta-blocker } \\
\text { use }\end{array}$ & NR & NR & 1.1 & $\begin{array}{l}0.92- \\
1.32\end{array}$ & OS & No & No & 6 \\
\hline 152 & $\mathrm{I}, 864$ & $\begin{array}{l}\text { Pre-diagnostic } \\
\text { beta-blocker } \\
\text { use }\end{array}$ & NR & NR & 1.05 & $\begin{array}{l}0.93- \\
1.18\end{array}$ & OS & No & No & 6 \\
\hline 196 & 1,929 & $\begin{array}{l}\text { Pre-diagnostic } \\
\text { beta-blocker } \\
\text { use }\end{array}$ & NR & NR & 1.01 & $\begin{array}{l}0.93- \\
1.11\end{array}$ & OS & No & No & 6 \\
\hline 163 & 1,705 & $\begin{array}{l}\text { Pre-diagnostic } \\
\text { beta-blocker } \\
\text { use }\end{array}$ & NR & NR & 1.18 & $\begin{array}{l}0.99- \\
1.40\end{array}$ & OS & No & No & 6 \\
\hline 1456 & $\mathrm{I}, 074$ & $\begin{array}{l}\text { Pre-diagnostic } \\
\text { beta-blocker } \\
\text { use }\end{array}$ & 79.2 & NR & 1.07 & $\begin{array}{l}0.96- \\
1.19\end{array}$ & OS & (1) & $\begin{array}{l}\text { Age at diagnosis, sex, year } \\
\text { of diagnosis, socioeconomic } \\
\text { status based on the place } \\
\text { of residence, Union for } \\
\text { International Cancer } \\
\text { Control (UICC) stage (I, II, } \\
\text { III, IV), cancer site (colon, } \\
\text { rectum/rectosigmoid), } \\
\text { surgery, chemotherapy, } \\
\text { radiotherapy, cancer, } \\
\text { cardiovascular disease, } \\
\text { cerebrovascular disease, } \\
\text { diabetes, hypertension, } \\
\text { time-dependent use of } \\
\text { NSAIDs, statins and diabetes } \\
\text { medication after diagnosis } \\
\text { and number of distinct ATC } \\
\text { classes prescribed during } \\
4 \text { months prior to diagnosis } \\
\text { (0, I-3, 4-5, 6+ distinct ATC } \\
\text { classes [first letter of the } \\
\text { ATC] dispensed during } \\
4 \text { months prior to diagnosis) }\end{array}$ & 7 \\
\hline 919 & 455 & $\begin{array}{l}\text { Post-diagnostic } \\
\text { beta blocker } \\
\text { use (time- } \\
\text { dependent) }\end{array}$ & 79.2 & NR & I.I & $\begin{array}{l}0.98- \\
1.23\end{array}$ & OS & Yes & $\begin{array}{l}\text { Age at diagnosis, sex, } \\
\text { year of diagnosis, socio- } \\
\text { economic status based on } \\
\text { the place of residence, Union } \\
\text { Internationale Contre le } \\
\text { Cancer (UICC) stage (I, II, } \\
\text { III, IV), cancer site (colon, } \\
\text { rectum/rectosigmoid), } \\
\text { surgery, chemotherapy, } \\
\text { radiotherapy, previous } \\
\text { cancer, cardiovascular } \\
\text { disease, cerebrovascular }\end{array}$ & 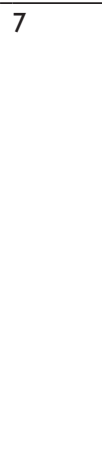 \\
\hline
\end{tabular}


Table I (Continued)

\begin{tabular}{|c|c|c|c|c|c|c|c|c|}
\hline Reference Study & Country & Duration & $\begin{array}{l}\text { Sample } \\
\text { size }\end{array}$ & $\begin{array}{l}\text { Median } \\
\text { age } \\
\text { (years) }\end{array}$ & $\begin{array}{l}\text { Study } \\
\text { design }\end{array}$ & Cancer type & Stage & $\begin{array}{c}\text { Surgery Beta-blocker } \\
\text { type }\end{array}$ \\
\hline
\end{tabular}

\begin{tabular}{|c|c|c|c|c|c|c|c|c|c|c|}
\hline 38 & $\begin{array}{l}\text { Livingstone } \\
\text { et al (20।3) }\end{array}$ & $\begin{array}{l}\text { Germany/ } \\
\text { The } \\
\text { Netherlands }\end{array}$ & & 709 & 6759 & PB cohort & Melanoma & NR & Mixed & $\begin{array}{l}\text { Mixed: beta I } \\
\text { selective (84\%); } \\
\text { non-selective } \\
(16 \%)\end{array}$ \\
\hline 39 & $\begin{array}{l}\text { Musselman } \\
\text { et al }(2014)\end{array}$ & Canada & $2002-2010$ & 66,889 & NR & PB cohort & Breast cancer & NR & Yes & NR \\
\hline 39 & $\begin{array}{l}\text { Musselman } \\
\text { et al }(2014)\end{array}$ & Canada & $2002-2010$ & 66,890 & NR & PB cohort & Lung cancer & NR & Yes & NR \\
\hline 39 & $\begin{array}{l}\text { Musselman } \\
\text { et al (2014) }\end{array}$ & Canada & $2002-2010$ & 66,891 & NR & PB cohort & $\begin{array}{l}\text { Colorectal } \\
\text { cancer }\end{array}$ & NR & Yes & NR \\
\hline 40 & $\begin{array}{l}\text { Parker et al } \\
(2017)\end{array}$ & USA & $2000-2010$ & 913 & 6567 & HB cohort & $\begin{array}{l}\text { Renal cell } \\
\text { carcinoma }\end{array}$ & $\begin{array}{l}\text { I/II } 5 \text { I.6\%, } \\
\text { III/IV 48.4\% }\end{array}$ & Yes & $\begin{array}{l}\text { Mixed: beta I } \\
\text { selective ( } 90 \%) ; \\
\text { non-selective } \\
(4 \%) \text {; alpha and } \\
\text { beta mixed }(6 \%)\end{array}$ \\
\hline 41 & $\begin{array}{l}\text { Sakellakis et al } \\
(20 \mid 4)\end{array}$ & Greece & $1983-2013$ & 610 & 6355 & HB cohort & Breast cancer & $\begin{array}{l}\text { I/II 73.6\%, } \\
\text { III/IV 26.4\% }\end{array}$ & Yes & Mixed \\
\hline 42 & $\begin{array}{l}\text { Shah et al } \\
(20 I I)\end{array}$ & UK & 1997-2009 & 3,462 & $\mathrm{HR}$ & PB cohort & Mixed cancer & NR & NR & $\begin{array}{l}\text { Mixed: beta } \\
\text { selective (83\%); } \\
\text { non-selective } \\
(17 \%)\end{array}$ \\
\hline
\end{tabular}




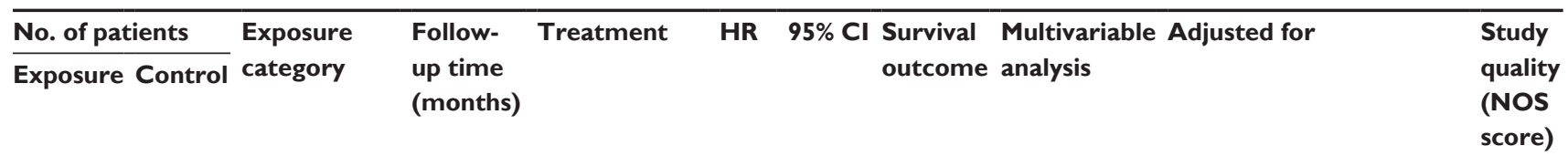

\begin{tabular}{|c|c|c|c|c|c|c|c|c|c|c|}
\hline & & & & & & & & & $\begin{array}{l}\text { disease, diabetes, } \\
\text { hypertension, time- } \\
\text { dependent use of NSAIDs, } \\
\text { statins and diabetes } \\
\text { medication after diagnosis } \\
\text { and number of distinct ATC } \\
\text { classes prescribed during } \\
\text { four months } \\
\text { prior to diagnosis (0, I-3, } \\
4-5,6+\text { distinct ATC classes } \\
\text { [first letter of the ATC] } \\
\text { dispensed during four } \\
\text { months prior to diagnosis) }\end{array}$ & \\
\hline 120 & 589 & $\begin{array}{l}\text { Post-diagnostic } \\
\text { beta-blocker } \\
\text { use (time- } \\
\text { dependent) }\end{array}$ & 39 & NR & 0.82 & $\begin{array}{l}0.55- \\
1.24\end{array}$ & OS & No & No & 6 \\
\hline 4,372 & 7,013 & NR & $\begin{array}{l}57.66 \\
30.5,43.1 \\
628.7, \\
\text { and } 53.46 \\
31.0\end{array}$ & NR & 0.99 & $\begin{array}{l}0.87- \\
1.13\end{array}$ & OS & No & No & 6 \\
\hline I,90I & 2,314 & NR & $\begin{array}{l}57.66 \\
30.5, \\
43.16 \\
28.7 \text {, and } \\
53.4631 .0\end{array}$ & NR & 1.06 & $\begin{array}{l}0.91- \\
1.24\end{array}$ & OS & No & No & 6 \\
\hline 22,170 & 30,118 & NR & $\begin{array}{l}57.66 \\
30.5, \\
43.16 \\
28.7 \text {, and } \\
53.4631 .0\end{array}$ & NR & 1.06 & $\begin{array}{l}0.99- \\
1.02\end{array}$ & OS & No & No & 6 \\
\hline \multirow[t]{2}{*}{104} & 809 & $\begin{array}{l}\text { Pre-diagnostic } \\
\text { beta-blocker } \\
\text { use }\end{array}$ & 98.4 & NR & 0.83 & $\begin{array}{l}0.59- \\
1.16\end{array}$ & OS & Yes & $\begin{array}{l}\text { Age at surgery, sex, } \\
\text { constitutional symptoms, } \\
\text { smoking history, eGFR } \\
\text { category, ECOG } \\
\text { performance status, } \\
\text { Charlson score, type of } \\
\text { surgery, tumor size, } 2010 \\
\text { PT classification, grade, } \\
\text { coagulative tumor necrosis }\end{array}$ & 7 \\
\hline & & & & & 0.78 & $\begin{array}{l}0.43- \\
1.41\end{array}$ & CSS & & & \\
\hline 47 & 430 & $\begin{array}{l}\text { Post-diagnostic } \\
\text { beta-blocker } \\
\text { use (time- } \\
\text { dependent) }\end{array}$ & 2448 & $\mathrm{CT}$ & 0.849 & $\begin{array}{l}0.537- \\
1.343\end{array}$ & DFS & No & No & 6 \\
\hline $\mathrm{I}, 406$ & 2,056 & $\begin{array}{l}\text { Pre-diagnostic } \\
\text { beta-blocker } \\
\text { use }\end{array}$ & NR & NR & 1.18 & $\begin{array}{l}1.04- \\
1.33\end{array}$ & OS & No & No & 6 \\
\hline
\end{tabular}


Table I (Continued)

\begin{tabular}{|c|c|c|c|c|c|c|c|c|c|c|}
\hline Reference & Study & Country & Duration & $\begin{array}{l}\text { Sample } \\
\text { size }\end{array}$ & $\begin{array}{l}\text { Median } \\
\text { age } \\
\text { (years) }\end{array}$ & $\begin{array}{l}\text { Study } \\
\text { design }\end{array}$ & Cancer type & Stage & Surgery & $\begin{array}{l}\text { Beta-blocker } \\
\text { type }\end{array}$ \\
\hline 43 & $\begin{array}{l}\text { Weberpals } \\
\text { et al (2017) }\end{array}$ & Holland & |998-20| | & 2,221 & 70.4 & PB cohort & Lung cancer & $\begin{array}{l}\text { I/II 24.1\%, } \\
\text { III/IV 75.9\% }\end{array}$ & $\begin{array}{l}\text { Mixed } \\
17.4 \%\end{array}$ & $\begin{array}{l}\text { Mixed: beta } \\
\text { selective (88\%); } \\
\text { non-selective } \\
(12 \%)\end{array}$ \\
\hline$\overline{43}$ & $\begin{array}{l}\text { Weberpals } \\
\text { et al (2017) }\end{array}$ & Holland & $|998-20| \mid$ & 2,221 & 70.4 & PB cohort & Lung cancer & $\begin{array}{l}\text { I/II 24.1\%, } \\
\text { III/IV 75.10\% }\end{array}$ & $\begin{array}{l}\text { Mixed } \\
17.5 \%\end{array}$ & $\begin{array}{l}\text { Mixed: beta } \\
\text { selective (88\%); } \\
\text { non-selective } \\
(13 \%)\end{array}$ \\
\hline
\end{tabular}

Abbreviations: NR, not reported; $\mathrm{PB}$, population-based; $\mathrm{HB}$, hospital-based; $\mathrm{RT}$, radiation therapy; $\mathrm{CT}$, chemotherapy; $\mathrm{ADT}$, androgen deprivation therapy; $\mathrm{CRT}$, concurrent chemoradiotherapy; IC, induction chemotherapy; GKRS, gamma knife radiosurgery; HRT, hormone replacement therapy; OS, overall survival; CSS, cancer-specific survival; DFS, disease-free survival; PFS, progression-free survival; RFS, recurrence-free survival; NOS, Newcastle-Ottawa Quality Assessment Scale; BMI, body mass index; IHD, ischemic heart disease; HT, hypertension; MBT, Metastatic brain tumors; ECOG, electrocorticogram; CVD, cardiovascular disease; GTN,GTV, gross tumor volume; RAM, Ramucirumab; PBO, Placebo; HHRR, hormonal receptor; THE, treatment emergent hypertension; ASA, American Standards Association; CAD, coronary artery disease; DM, diabetes mellitus; FIGO, International Federation of Gynecology and Obstetrics; eGFR, epidermal growth factor receptor; ATC, Anatomical Therapeutic Chemical; CCI, Charlson comorbidity index; DMFS, distant metastasis-free survivall; pT, primary tumour.

the combined HR of 0.99 (95\% CI: $0.94-1.05, P=0.807$, Figure 2B) showed that beta-blocker use was also not correlated with all-cause mortality.

\section{Meta-analysis of cancer-specific survival}

Thirteen studies presented the data concerning the association between beta-blocker use and CSS (Figure 2C). We calculated that beta-blocker use was significantly correlated with long CSS, with a pooled HR of 0.78 (95\% CI: $0.65-0.95$, $P=0.012$ ) by using a random-effects model.

\section{Meta-analysis of disease-free survival}

Four studies reported the data on beta-blocker use and DFS outcome. The pooled HR was 0.59 (95\% CI: 0.30-1.17, $P=0.134$, Figure 2D) with significant heterogeneity between studies $\left(I^{2}=89.5 \%, P<0.001\right)$, which demonstrated that betablocker use was also prominently not related to DFS.

\section{Meta-analysis of progression-free survival}

The data on beta-blocker use and PFS outcome was presented in six studies. Meta-analysis adopting the fixed-effects model revealed that beta-blocker use was not associated with PFS (HR=0.90, 95\% CI: 0.79-1.02, $P=0.087$, Figure 2E) and exhibited no heterogeneity $\left(I^{2}=0.00 \%, P=0.603\right)$.

\section{Meta-analysis of recurrence-free survival}

Four studies provided sufficient data on beta-blocker use and RFS outcome. The pooled HR was 0.99 (95\% CI: 0.76-1.28, $P=0.944$, Figure $2 \mathrm{~F}$ ) by a random-effects model. Beta-blocker use was also significantly not related to RFS.

\section{Subgroup analysis}

To deeply explore the relationship between beta-blocker use and OS, we performed subgroup analysis based on ethnicity, duration of drug use, cancer stage, sample size, beta-blocker type, chronological order of drug use, and different types of cancers. The median values of original data from included studies in "duration of drug use" and "sample size" were chosen as cut-off values to divide our subgroups. The results are summarized in Table 2, with the corresponding forest plots presented in Figure S1.

The subgroups of sample size and ethnicity demonstrated no significant effect of beta-blocker use on OS. Similarly, beta-blocker showed no obvious impact on OS for patients with duration of drug use more than 2 years $(\mathrm{HR}=1.03,95 \%$ CI: $0.93-1.14, P=0.617$ ) or patients with duration of drug use less than 2 years (HR=1.01, 95\% CI: 0.91-1.11, $P=0.897)$. Additionally, the subgroup analysis indicated that the administration of beta-blockers had no relationship with longer OS when the meta-analysis was restricted to patients with cancer in I/II stage ( $\mathrm{HR}=0.97,95 \% \mathrm{CI}: 0.89-1.06, P=0.507)$ or cancer in III/IV stage $(\mathrm{HR}=1.04,95 \% \mathrm{CI}: 0.94-1.14, P=0.468)$. In addition, the studies using selective beta-blocker $(\mathrm{HR}=0.93$, 95\% CI: $0.83-1.05, P=0.243$ ) and non-selective beta-blocker $(\mathrm{HR}=1.04,95 \% \mathrm{CI}: 0.89-1.22, P=0.596)$ were found to have no effect on OS. However, beta-blocker showed a more positive effect on OS for patients with time-fixed post-diagnostic beta-blocker use ( $\mathrm{HR}=0.65,95 \% \mathrm{CI}$ : $0.43-0.99, P=0.046)$ than pre-diagnostic beta-blocker use $(\mathrm{HR}=1.03,95 \% \mathrm{CI}$ : $0.95-1.11, P=0.493)$ and time-dependent post-diagnostic beta-blocker use $(\mathrm{HR}=0.87,95 \% \mathrm{CI}$ : $0.59-1.30, P=0.508)$. 


\begin{tabular}{|c|c|c|c|c|c|c|c|c|c|c|}
\hline \multicolumn{2}{|c|}{ No. of patients } & \multirow{2}{*}{$\begin{array}{l}\text { Exposure } \\
\text { category }\end{array}$} & \multirow{2}{*}{$\begin{array}{l}\text { Follow- } \\
\text { up time } \\
\text { (months) }\end{array}$} & \multirow{2}{*}{ Treatment } & \multirow[t]{2}{*}{ HR } & \multirow[t]{2}{*}{$95 \% \mathrm{Cl}$} & \multirow{2}{*}{$\begin{array}{l}\text { Survival } \\
\text { outcome }\end{array}$} & \multirow{2}{*}{$\begin{array}{l}\text { Multivariable } \\
\text { analysis }\end{array}$} & \multirow{2}{*}{ Adjusted for } & \multirow{2}{*}{$\begin{array}{l}\text { Study } \\
\text { quality } \\
\text { (NOS } \\
\text { score) }\end{array}$} \\
\hline Exposure & Control & & & & & & & & & \\
\hline $\mathrm{I}, 107$ & 1,114 & $\begin{array}{l}\text { Pre-diagnostic } \\
\text { beta-blocker } \\
\text { use }\end{array}$ & 78 & NR & 1 & $\begin{array}{l}0.92- \\
1.08\end{array}$ & OS & Yes & $\begin{array}{l}\text { Comorbidities, time-varying } \\
\text { treatment, and distinct } \\
\text { numbers of medications used }\end{array}$ & 7 \\
\hline $\mathrm{I}, 224$ & 997 & $\begin{array}{l}\text { Post-diagnostic } \\
\text { beta-blocker } \\
\text { use (time- } \\
\text { dependent) }\end{array}$ & 78 & NR & 1.03 & $\begin{array}{l}0.94- \\
1.11\end{array}$ & OS & Yes & $\begin{array}{l}\text { Comorbidities, time-varying } \\
\text { treatment, and distinct } \\
\text { numbers of medications used }\end{array}$ & 7 \\
\hline
\end{tabular}

Analysis according to cancer type showed predominantly longer OS in ovarian cancer (HR $=0.59,95 \%$ CI: $0.36-0.96$, $P=0.034$ ), pancreatic cancer (HR $=0.85,95 \%$ CI: $0.75-0.97$, $P=0.014$ ), and melanoma (HR=0.81, 95\% CI: 0.67-0.97, $P=0.026)$, but no effects on lung cancer $(\mathrm{HR}=1,95 \%$ CI: $0.96-1.05, P=0.818)$, breast cancer (HR $=0.97,95 \%$ CI: $0.78-1.21, P=0.783)$, colorectal cancer $(\mathrm{HR}=1.16$; $95 \%$ CI: $0.84-1.61, P=0.353)$, and mixed cancer (HR=1.00; $95 \%$ CI: $0.83-1.21, P=0.974)$. Owing to the small numbers of studies and lack of information, subgroup analyses were not performed on other survival outcomes.

\section{Sensitivity analysis}

Sensitivity analysis was conducted on different survival outcomes. The meta-analyses of beta-blockers and survival were performed by removing a single study in turn. After removing the study results, the comprehensive estimation direction and amplitude of OS, all-cause mortality, CSS, DFS, PFS, and RFS were not significantly changed, indicating that the reliability of the meta-analysis was good and the results were not affected by any research (Figure 3 ). In addition, sensitivity analyses were also conducted in those studies whose HR and $95 \% \mathrm{CI}$ values were presented in original articles (not calculated from the Kaplan-Meier plots) (Figure S2) and whose NOS score was $\geq 7$ (Figure S3). These factors did not affect the main results.

\section{Publication bias}

The funnel plot revealed no evidence of publication bias in the meta-analysis of beta-blocker use and OS (Figure 4A, Egger's test: $P$-value $=0.358 ;$ Begg's test: $P$-value $=0.115)$. There was no potential publication bias on beta-blocker use and all-cause mortality as well (Figure 4B, Egger's test: $P$-value $=0.261$; Begg's test: $P$-value $=0.260$ ). Besides, there was also no potential publication bias on beta-blocker use, CSS, DFS, PFS, and RFS of cancer patients (Figure 4C-F).

\section{Meta-regression}

The meta-regression analysis was performed to investigate the effects of various cohort study characteristics on the study estimates of the HRs. We grouped the studies according to specific characteristics, the size of sample, the sex of patients, the cancer sites, study duration, and study quality. There was no inverse association between sample size $(P=0.892)$, sex of the patients $(P=0.135)$, cancer sites $(P=0.364)$, study duration $(P=0.076)$, and study quality $(P=0.571)$. Because of the lack of information, meta-regression was not performed on other survival outcomes.

\section{Discussion}

This meta-analysis summarizes 36 currently published studies examining the association between beta-blocker use and prognosis of cancer across a wide range of geographic regions and cancer types. Overall, the administration of beta-blocker was not associated with OS, all-cause mortality, DFS, PFS and RFS of cancer patients. However, beta-blocker use was significantly correlated with long CSS (HR $=0.78,95 \%$ CI: 0.65-0.95). Since the patients included in the clinical trials differed in stages, therapies, and so on, the heterogeneity was inescapable. Then we conducted subgroup analysis. 
A

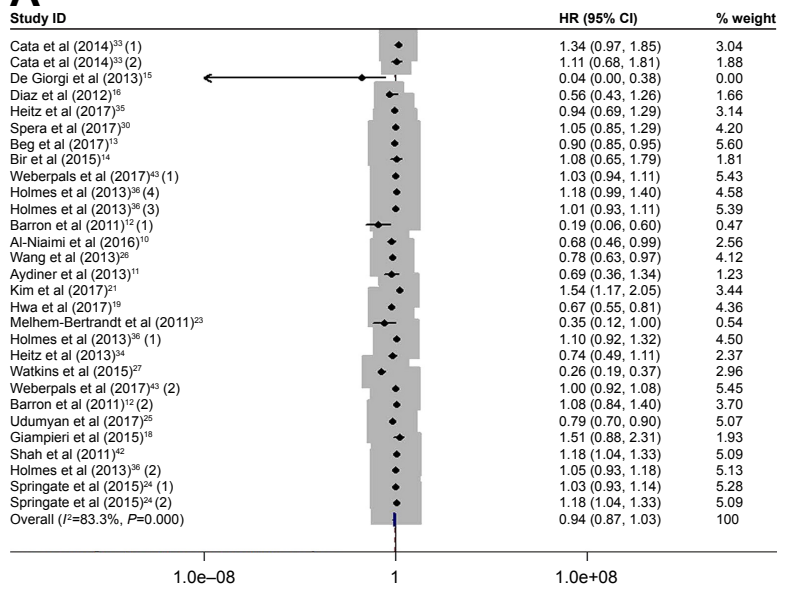

C

Study ID

Hwa et al (2017)

Assayag et al (2014) $)^{32}$

Melhem-Bertrandt et al (2011)

Lemeshow et al (2011) ${ }^{22}$

Kim et al (2017)

Grytli et al (2013)

Grytli et al (2014)

Ganz et al (2011)

Barron et al (2011) 12

Watkins et al (2015)

Udumyan et al (2017)

Parker et al $(2017)^{40}$

Jansen et al (2014)

Overall $\left(I^{2}=84.5 \%, P=0.000\right)$

0.17

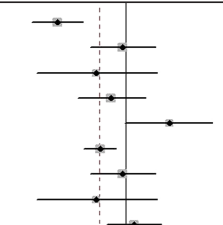

$0.76(0.44,1.33) \quad 5.45$

$0.87(0.64,1.20) 7.95$

$1.49(0.99,2.22) 6.96$

$0.79(0.68,0.91) \quad 9.60$

$0.97(0.72,1.31) 8.11$

$0.76(0.44,1.33) \quad 5.45$

$1.08(0.84,1.39) 8.62$

$0.24(0.17,0.34) \quad 7.59$

$(0.69,0.87) \quad 9.80$

$0.78(0.43,1.41) \quad 5.09$

$0.93(0.71,1.21) 8.47$

$0.78(0.65,0.95) \quad 100$

5.88

E

Study ID

HR $(95 \% \mathrm{Cl}) \quad \%$ weight

Heitz et al $(2017)^{35}$

Spera et al $(2017)^{30}$

Giampieri et al (2015)

Diaz et al (2012) ${ }^{16}$

Heitz et al (2013) $)^{34}$

Wang et al $(2013)^{26}$

Overall $\left(I^{2}=0.0 \%, P=0.603\right)$

0.48

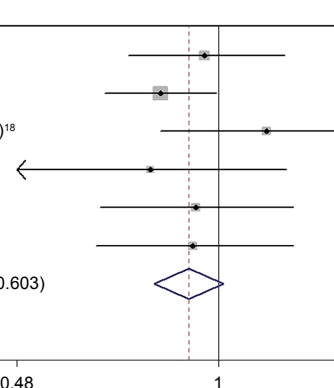

$0.95(0.72,1.27) 19.44$

$0.81(0.66,0.99) 38.08$

$1.19(0.81,1.72) 11.04$

$0.78(0.48,1.28) 6.51$

$0.92(0.65,1.31) 12.75$

$0.91(0.64,1.31) 12.20$

$0.90(0.79,1.02) 100$
B

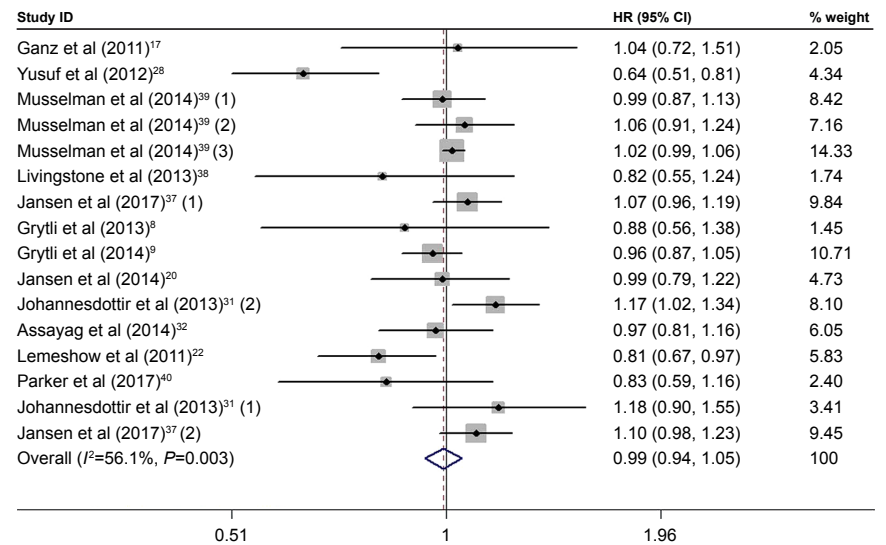

D

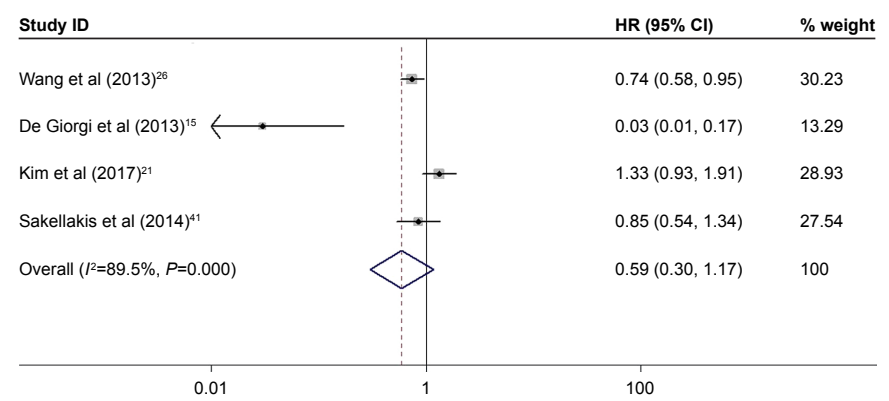

$\mathbf{F}$

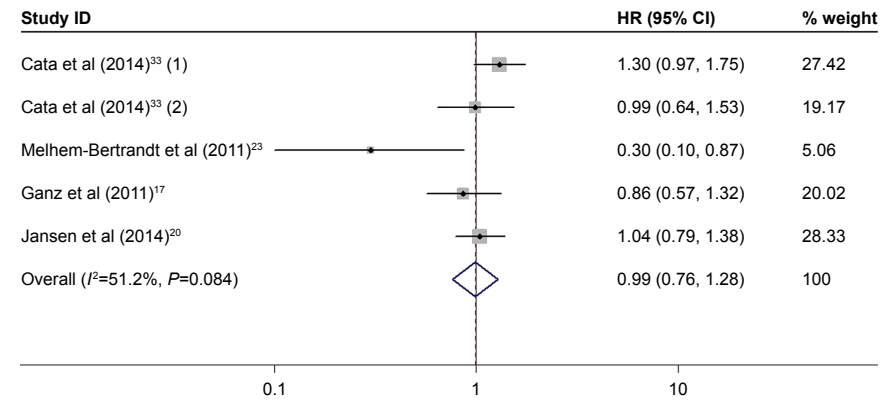

Figure 2 Forest plots showing the effects of beta-blocker use on OS (A), all-cause mortality (B), CSS (C), DFS (D), PFS (E), and RFS (F).

Notes: Weights are from random-effects analysis. The numbers in parentheses indicate the different included studies in the same year.

Abbreviations: OS, overall survival; CSS, cancer-specific survival; DFS, disease-free survival; PFS, progression-free survival; RFS, recurrence-free survival.

Among the cancer types, positive associations between beta-blocker use and cancer prognosis were observed in breast cancer, pancreatic cancer, and melanoma, but could not be detected in lung cancer, ovarian cancer, colorectal cancer, and mixed cancer. Interestingly, beta-blocker use is associated with improved survival only among patients with ovarian cancer, pancreatic cancer, and melanoma. However, the results should be interpreted carefully because the number of studies on these three cancers was small. In addition, the results showed that beta-blockers prolonged OS for patients with time-fixed post-diagnostic beta-blocker use. Generally, the subgroups of cancer stage, beta-blocker type, cumulative beta-blocker use, sample size, and ethnicity demonstrated no significant effect of beta-blocker on longer OS. Hence, we did not find a beneficial effect of beta-blocker use on cancer survival.

To our knowledge, this meta-analysis is the fourth one to be conducted on beta-blocker use and prognosis in various cancers. Indeed, this analysis objectively confirmed the latest development in this topic. All the previous three articles drew a conclusion that beta-blocker use could prolong the survival of cancer patients, ${ }^{44-46}$ but our current analysis showed an opposite conclusion that there is generally no relationship between beta-blocker use and cancer prognosis. 
Table 2 Summary of the subgroup analysis results of beta-blocker use and OS

\begin{tabular}{|c|c|c|c|c|c|c|c|}
\hline \multirow[t]{2}{*}{ Variables } & \multirow{2}{*}{$\begin{array}{l}\text { Number } \\
\text { of studies }\end{array}$} & \multirow{2}{*}{$\begin{array}{l}\text { Number } \\
\text { of patients }\end{array}$} & \multirow[t]{2}{*}{ Model } & \multicolumn{2}{|l|}{ Outcome (OS) } & \multicolumn{2}{|c|}{ Heterogeneity } \\
\hline & & & & HR (95\% Cl) & $P$-value & $I^{2}(\%)$ & $P$-value \\
\hline \multicolumn{8}{|l|}{ Ethnicity } \\
\hline Non-Europeans & 16 & 30,607 & $\mathrm{R}$ & $0.90(0.78-1.02)$ & 0.106 & 87.2 & $<0.001$ \\
\hline Europeans & 8 & 12,182 & $\mathrm{R}$ & $1.00(0.89-I .12)$ & 0.958 & 72.2 & 0.001 \\
\hline \multicolumn{8}{|l|}{ Duration of drug use } \\
\hline$>2$ years & 6 & 8,899 & $\mathrm{~F}$ & $1.03(0.93-1.14)$ & 0.617 & 0.0 & 0.576 \\
\hline$<2$ years & 6 & 10,812 & $\mathrm{R}$ & I.0I (0.9I-I.II) & 0.897 & 54.7 & 0.051 \\
\hline \multicolumn{8}{|l|}{ Cancer stage } \\
\hline $\mathrm{I} / \mathrm{II}$ & 11 & 2,870 & $\mathrm{~F}$ & $0.97(0.89-1.06)$ & 0.507 & 15.6 & 0.295 \\
\hline III/IV & 13 & 4,835 & $\mathrm{R}$ & $1.04(0.94-1.14)$ & 0.468 & 59.1 & 0.003 \\
\hline \multicolumn{8}{|l|}{ Sample size } \\
\hline$>1,500$ & 15 & 65,834 & $\mathrm{R}$ & I.0I (0.94-I.08) & 0.783 & 76.7 & $<0.001$ \\
\hline$<1,500$ & 18 & 11,839 & $\mathrm{R}$ & $0.81(0.66-1.00)$ & 0.053 & 83.5 & $<0.001$ \\
\hline \multicolumn{8}{|l|}{ Beta-blocker type } \\
\hline Non-selective & 12 & $17,7 \mid 4$ & $\mathrm{R}$ & $1.04(0.89-1.22)$ & 0.596 & 75.7 & $<0.001$ \\
\hline Selective & 10 & $17,7 \mid 4$ & $\mathrm{R}$ & $0.93(0.83-1.05)$ & 0.243 & 83.5 & $<0.001$ \\
\hline \multicolumn{8}{|l|}{ Chronological order of drug use } \\
\hline Pre-diagnostic beta-blocker use & 13 & 55,710 & $\mathrm{R}$ & $\mathrm{I} .03(0.95-1.1 \mathrm{I})$ & 0.493 & 74.7 & $<0.001$ \\
\hline Post-diagnostic beta-blocker use (time-fixed) & 7 & 6,372 & $\mathrm{R}$ & $0.65(0.43-0.99)$ & 0.046 & 91.0 & $<0.001$ \\
\hline Post-diagnostic beta blocker use (time-dependent) & 2 & 2,406 & $\mathrm{R}$ & $0.87(0.59-1.30)$ & 0.508 & 76.8 & 0.038 \\
\hline \multicolumn{8}{|l|}{ Cancer type } \\
\hline Lung cancer & 7 & 10,189 & $\mathrm{~F}$ & $1.01(0.96-1.05)$ & 0.818 & 40.1 & 0.124 \\
\hline Melanoma & 2 & 4,910 & $\mathrm{~F}$ & $0.8 \mathrm{I}(0.67-0.97)$ & 0.026 & 0.0 & 0.892 \\
\hline Mixed cancer & 4 & 21,494 & $\mathrm{R}$ & $1.00(0.83-1.21)$ & 0.974 & 87.7 & $<0.001$ \\
\hline Colorectal cancer & 2 & 4,202 & $\mathrm{R}$ & $1.16(0.84-1.61)$ & 0.353 & 51.3 & 0.152 \\
\hline Ovarian cancer & 5 & 3,140 & $\mathrm{R}$ & $0.59(0.36-0.96)$ & 0.034 & 88.0 & $<0.001$ \\
\hline Breast cancer & 6 & 16,637 & $\mathrm{R}$ & $0.97(0.78-1.2 I)$ & 0.783 & 61.20 & 0.024 \\
\hline Pancreatic cancer & 2 & 16,096 & $\mathrm{R}$ & $0.85(0.75-0.97)$ & 0.014 & 71.10 & 0.063 \\
\hline
\end{tabular}

Abbreviations: $\mathrm{F}$, fixed-effects model; R, random-effects model; OS, overall survival.

We then hypothesize some possible reasons for this conclusion. Preclinical studies have suggested that $\beta$-blockers play an anti-cancer role in multiple kinds of cancers by targeting at $\beta$-adrenergic signaling pathway. ${ }^{47,48} \beta$-blockers can inhibit multiple processes of tumor progression and metastasis, including the inhibition of tumor cell proliferation, migration, invasion, as well as resistance to tumor angiogenesis and metastasis. ${ }^{3}$ Although the basic research may be effective, it is not recommended for speculating on the clinical survival of cancer patients due to the current evidence of evidencebased medicine. Beta-blocker is not a necessary medication for general adjuvant chemotherapy in cancer patients. ${ }^{49}$

Since cardiovascular diseases are common in the population, cancer patients frequently receive cardiovascular medications, including beta-blockers, ${ }^{2}$ but beta-blockers might not be recommended for chemotherapy in the absence of other indications. Further studies should be done to investigate the relationship between cancer survival and beta-blocker use in cancer patients without cardiovascular disease. Additionally, different effects in different cancers might have contributed to the lack of a discernible relationship between beta-blockers and OS of various cancers in the current studies. To find out the actual concrete relationship between the two, further analysis can be confined to beta-blocker use and one specific cancer based on a large enough population. Besides, beta-blockers themselves might have some undefined side effects on other organ systems, which might lead to cancer progression. ${ }^{50}$

However, there are still several limitations in this study. First, the studies included in this analysis were all cohort studies or case-control studies, as there were no RCTs yet investigating this topic. Second, while sensitivity analysis supported the stability of our results and a relatively large number of studies were included, we should still carefully interpret the results. The heterogeneity found in the study may be attributed to the multivariable influence factors in some studies. Third, the power of Begg's and Egger's tests to detect bias will be low with small number of studies, and when the between-study heterogeneity is large, none of the bias detection tests work well. Fourth, the dose-response analyses were not carried out due to a limited amount of literature. 
A

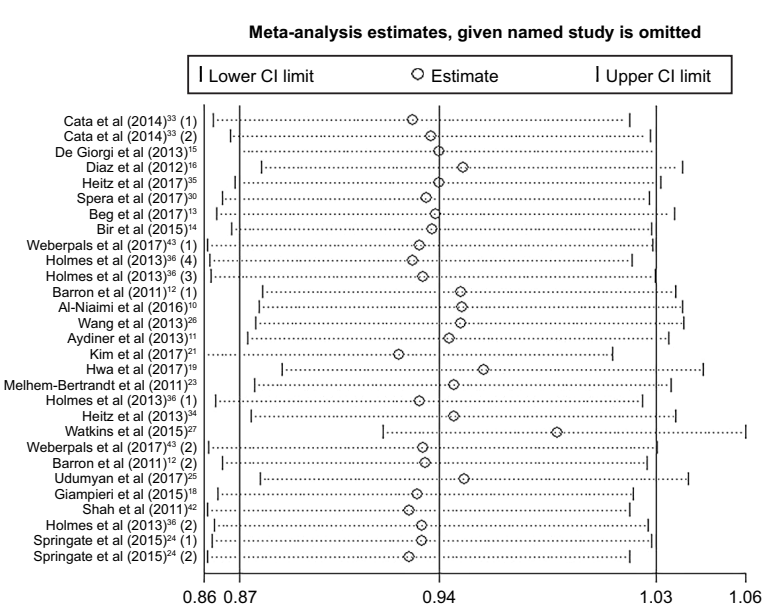

C

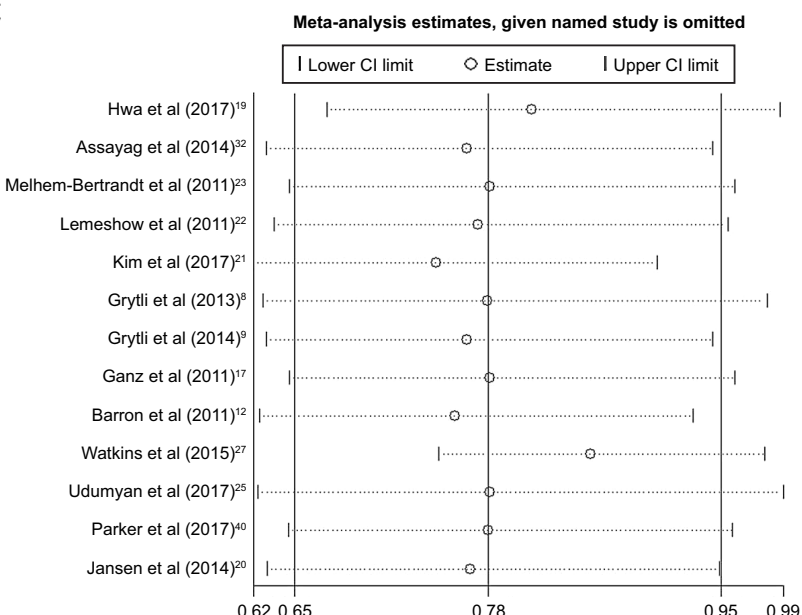

E
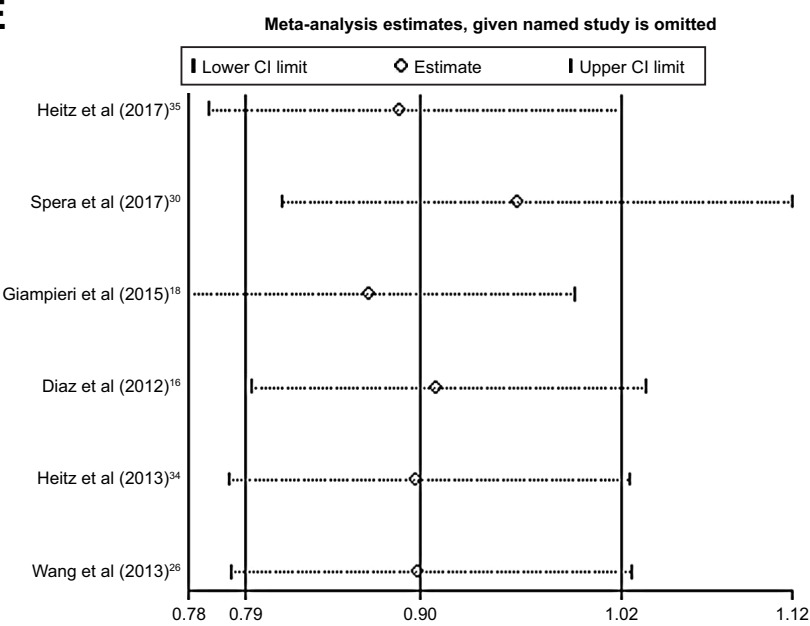

B

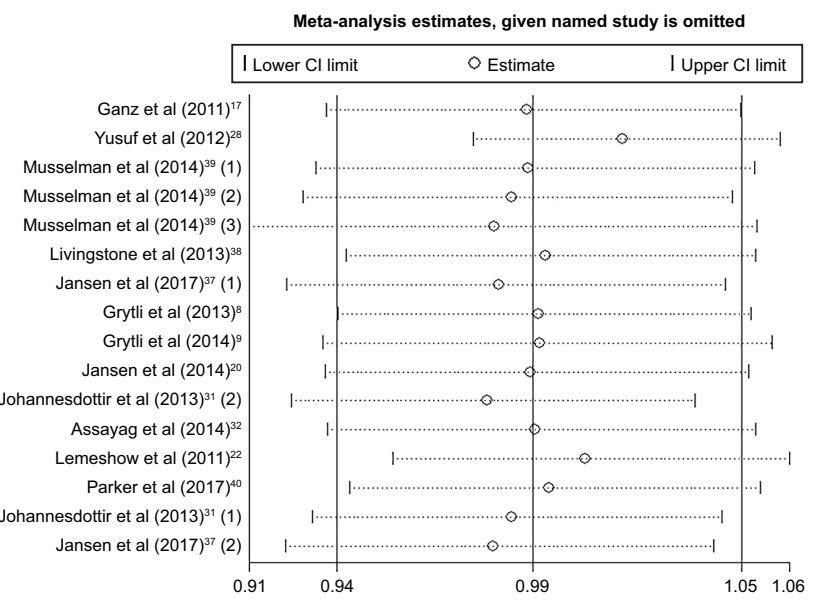

D

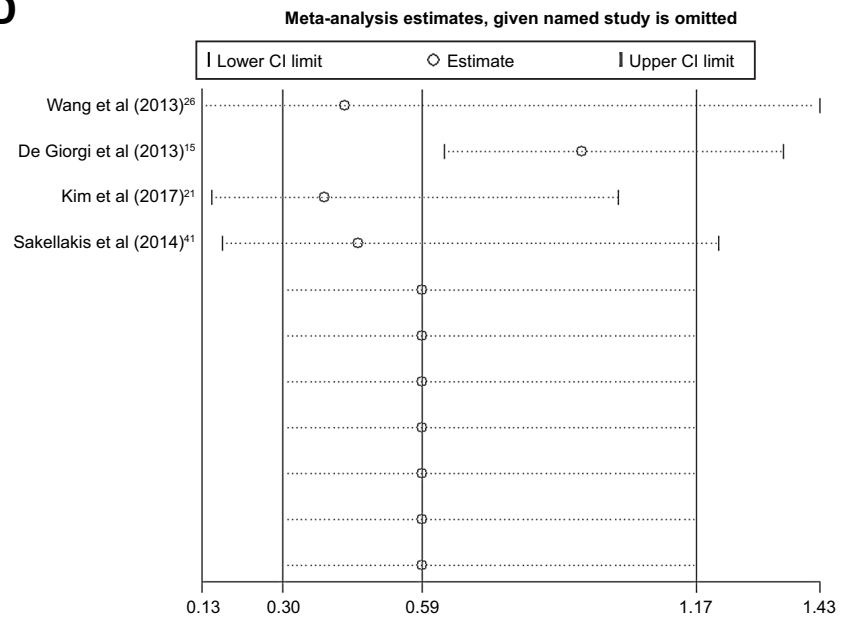

$\mathbf{F}$

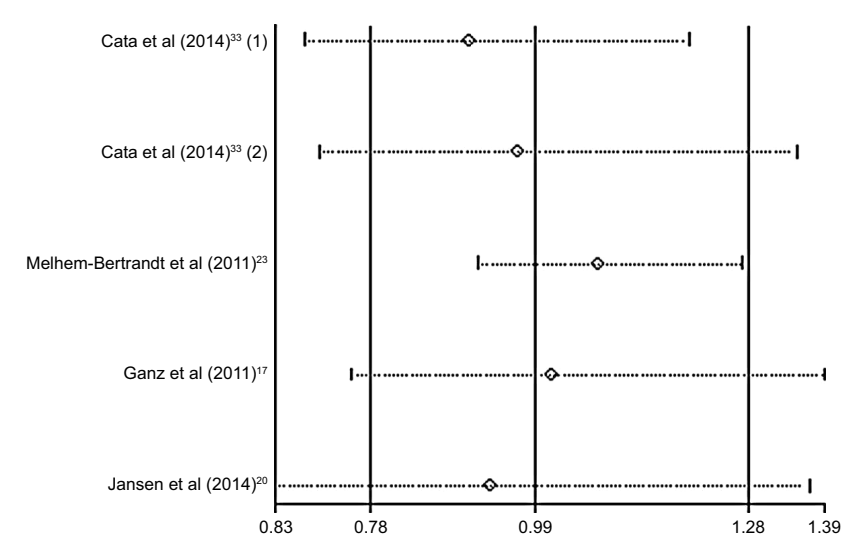

Figure 3 Sensitivity analysis of beta-blocker use on OS (A), all-cause mortality (B), CSS (C), DFS (D), PFS (E), and RFS (F).

Abbreviations: OS, overall survival; CSS, cancer-specific survival; DFS, disease-free survival; PFS, progression-free survival; RFS, recurrence-free survival.

Despite the limitations, there are several strengths in our study compared with previous meta-analyses. First, our current analysis showed a completely different main conclusion from the previous meta-analyses that there was no relationship between beta-blocker use and cancer prognosis.
Second, we separated all-cause mortality from OS to make the analysis more precise. Third, we included 36 studies involving 319,006 patients, which was a larger number of patients than previous meta-analyses. Fourth, we discussed almost all variables that could describe the outcome of 

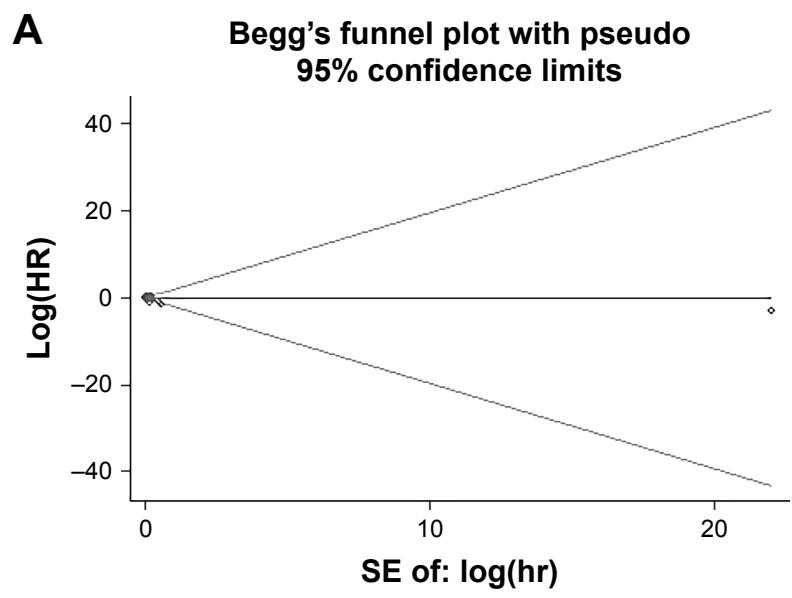

C Begg's funnel plot with pseudo

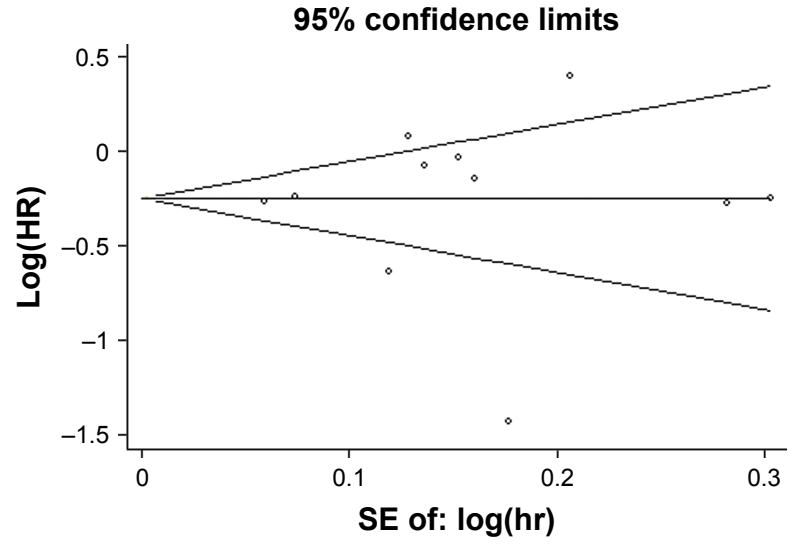

$\mathbf{E}$

Begg's funnel plot with pseudo 95\% confidence limits

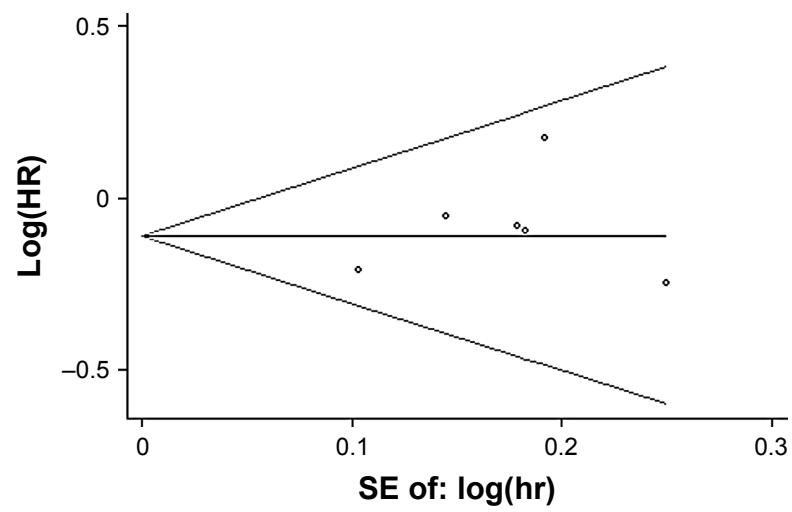

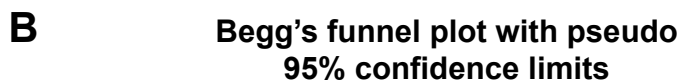
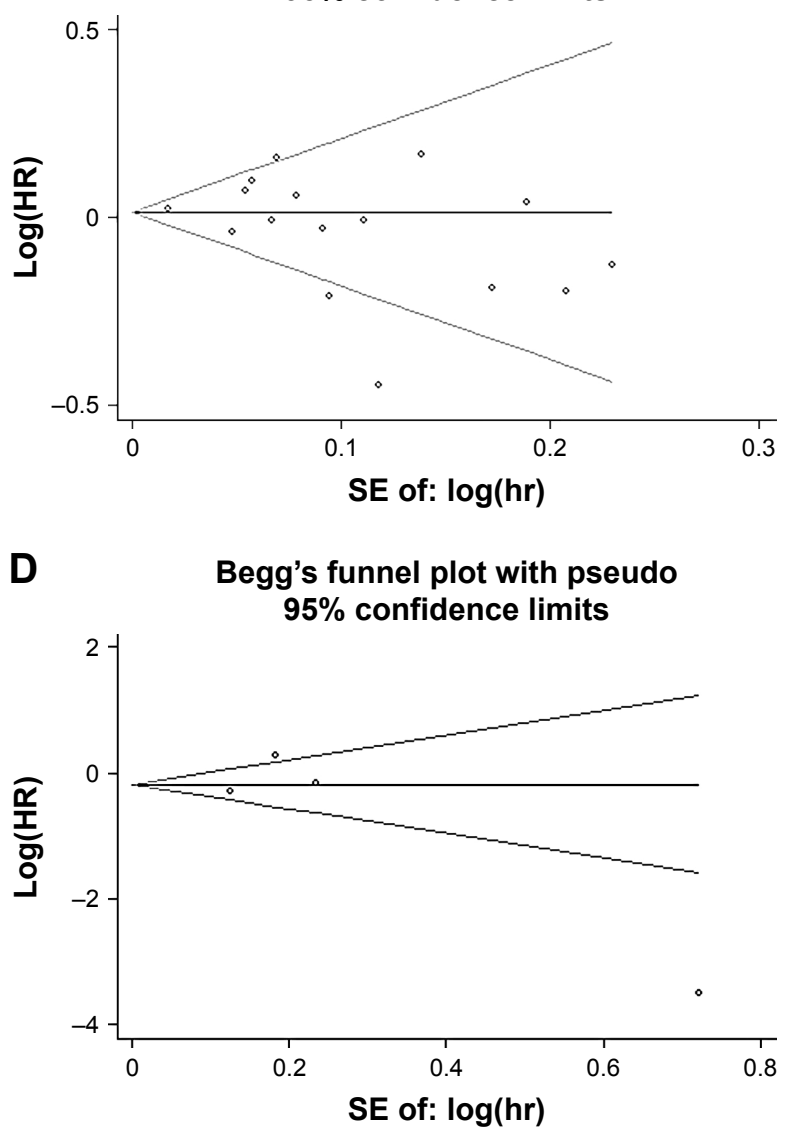

$\mathbf{F}$

Begg's funnel plot with pseudo $95 \%$ confidence limits

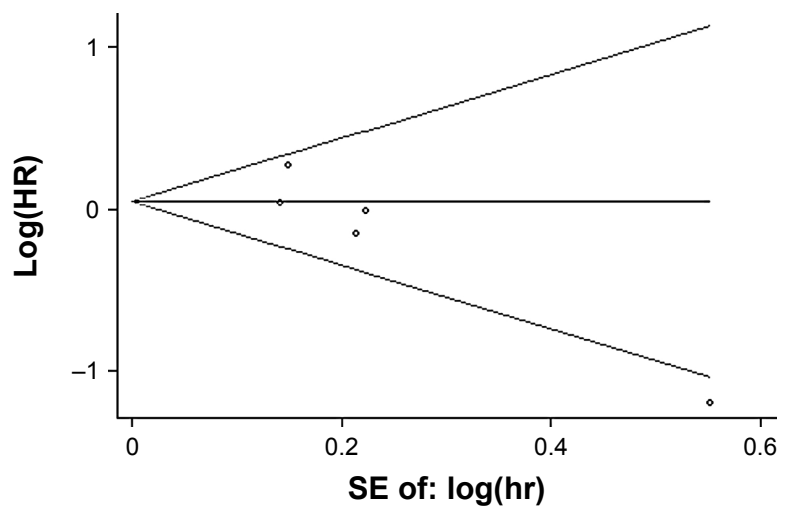

Figure 4 Funnel plot of Begg's test of beta blocker use on OS (A), all-cause mortality (B), CSS (C), DFS (D), PFS (E), and RFS (F).

Abbreviations: OS, overall survival; CSS, cancer-specific survival; DFS, disease-free survival; PFS, progression-free survival; RFS, recurrence-free survival; SE, standard error.

survival, including OS, all-cause mortality, CSS, DFS, PFS, and RFS.

\section{Conclusion}

The beta-blocker administration is not associated with cancer prognosis except for the positive effect on long CSS. Moreover, there are apparent protective effects of beta-blocker use in ovarian cancer, pancreatic cancer, and melanoma. We need more high-quality studies, such as RCTs, to confirm this conclusion in the future.

\section{Disclosure}

The authors report no conflicts of interest in this work. 


\section{References}

1. Siegel RL, Miller KD, Jemal A. Cancer Statistics, 2017. CA Cancer J Clin. 2017;67:7-30.

2. Wiysonge CS, Bradley HA, Volmink J, et al. Beta-blockers for hypertension. Cochrane Database Syst Rev. 2017;1:CD002003.

3. Creed SJ, Le CP, Hassan M, et al. Beta2-adrenoceptor signaling regulates invadopodia formation to enhance tumor cell invasion. Breast Cancer Res. 2015;17:145.

4. Nagaraja AS, Sadaoui NC, Lutgendorf SK, Ramondetta LM, Sood AK. Beta-blockers: a new role in cancer chemotherapy? Expert Opin Investig Drugs. 2013;22:1359-1363.

5. Tierney JF, Stewart LA, Ghersi D, Burdett S, Sydes MR. Practical methods for incorporating summary time-to-event data into meta-analysis. Trials. 2007;8:16.

6. Parmar MK, Torri V, Stewart L. Extracting summary statistics to perform meta-analyses of the published literature for survival endpoints. Stat Med. 1998;17:2815-2834.

7. Harris R, Bradburn M, Deeks J, et al. METAN: stata module for fixed and random effects meta-analysis. Statistical Software Components. 2010;8(1):3-28.

8. Grytli HH, Fagerland MW, Fossa SD, Tasken KA, Haheim LL. Use of beta-blockers is associated with prostate cancer-specific survival in prostate cancer patients on androgen deprivation therapy. Prostate. 2013;73:250-260.

9. Grytli HH, Fagerland MW, Fossa SD, Tasken KA. Association between use of beta-blockers and prostate cancer-specific survival: a cohort study of 3561 prostate cancer patients with high-risk or metastatic disease. Eur Urol. 2014;65:635-641.

10. Al-Niaimi A, Dickson EL, Albertin C, et al. The impact of perioperative beta blocker use on patient outcomes after primary cytoreductive surgery in high-grade epithelial ovarian carcinoma. Gynecol Oncol. 2016;143:521-525.

11. Aydiner A, Ciftci R, Karabulut S, Kilic L. Does beta-blocker therapy improve the survival of patients with metastatic non-small cell lung cancer? Asian Pac J Cancer Prev. 2013;14:6109-6114.

12. Barron TI, Connolly RM, Sharp L, Bennett K, Visvanathan K. Beta blockers and breast cancer mortality: a population-based study. J Clin Oncol. 2011;29:2635-2644.

13. Beg MS, Gupta A, Sher D, et al. Impact of concurrent medication use on pancreatic cancer survival-SEER-medicare analysis. Am J Clin Oncol. Epub 2017 Jan 10

14. Bir SC, Kalakoti P, Ahmed O, Bollam P, Nanda A. Elucidating the role of incidental use of beta-blockers in patients with metastatic brain tumors in controlling tumor progression and survivability. Neurol India. 2015;63:19-23

15. De Giorgi V, Gandini S, Grazzini M, Bollam P, Nanda A. Effect of beta-blockers and other antihypertensive drugs on the risk of melanoma recurrence and death. Mayo Clin Proc. 2013;88:1196-1203.

16. Diaz ES, Karlan BY, Li AJ. Impact of beta blockers on epithelial ovarian cancer survival. Gynecol Oncol. 2012;127:375-378.

17. Ganz PA, Habel LA, Weltzien EK, Caan BJ, Cole SW. Examining the influence of beta blockers and ACE inhibitors on the risk for breast cancer recurrence: results from the LACE cohort. Breast Cancer Res Treat. 2011;129:549-556.

18. Giampieri R, Scartozzi M, Del Prete M, et al. Prognostic value for incidental antihypertensive therapy with beta-blockers in metastatic colorectal cancer. Medicine (Baltimore). 2015;94:e719.

19. Hwa YL, Shi Q, Kumar SK, et al. Beta-blockers improve survival outcomes in patients with multiple myeloma: a retrospective evaluation. Am J Hematol. 2017;92:50-55.

20. Jansen L, Hoffmeister M, Arndt V, Chang-Claude J, Brenner H. Stagespecific associations between beta blocker use and prognosis after colorectal cancer. Cancer. 2014;120:1178-1186.

21. Kim SA, Moon H, Roh JL, et al. Postdiagnostic use of beta-blockers and other antihypertensive drugs and the risk of recurrence and mortality in head and neck cancer patients: an observational study of 10,414 person-years of follow-up. Clin Transl Oncol. 2017;19:826-833.
22. Lemeshow S, Sorensen HT, Phillips G, et al. Beta-blockers and survival among Danish patients with malignant melanoma: a populationbased cohort study. Cancer Epidemiol Biomarkers Prev. 2011;20: 2273-2279.

23. Melhem-Bertrandt A, Chavez-Macgregor M, Lei X, et al. Beta-blocker use is associated with improved relapse-free survival in patients with triple-negative breast cancer. J Clin Oncol. 2011;29:2645-2652.

24. Springate DA, Ashcroft DM, Kontopantelis E, Doran T, Ryan R, Reeves D. Can analyses of electronic patient records be independently and externally validated? Study 2 - the effect of beta-adrenoceptor blocker therapy on cancer survival: a retrospective cohort study. BMJ Open. 2015;5:e007299.

25. Udumyan R, Montgomery S, Fang F, et al. Beta-blocker drug use and survival among patients with pancreatic adenocarcinoma. Cancer Res. 2017;77:3700-3707.

26. Wang HM, Liao ZX, Komaki R, et al. Improved survival outcomes with the incidental use of beta-blockers among patients with non-smallcell lung cancer treated with definitive radiation therapy. Ann Oncol. 2013;24:1312-1319.

27. Watkins JL, Thaker PH, Nick AM, et al. Clinical impact of selective and nonselective beta-blockers on survival in patients with ovarian cancer. Cancer. 2015;121:3444-3451.

28. Yusuf SW, Daraban N, Abbasi N, Lei X, Durand JB, Daher IN. Treatment and outcomes of acute coronary syndrome in the cancer population. Clin Cardiol. 2012;35:443-450.

29. Botteri E, Munzone E, Rotmensz N, et al. Therapeutic effect of b-blockers in triple-negative breast cancer postmenopausal women. Breast Cancer Res Treat. 2013;140:567-575.

30. Spera G, Fresco1 R, Fung H, et al. Beta blockers and improved progression free survival in patients with advanced HER2 negative breast cancer: a retrospective analysis of the ROSE/TRIO-012 study. Ann Oncol. 2017;28(8):1836-1841.

31. Johannesdottir SA, Schmidt M, Phillips G, et al. Use of ß-blockers and mortality following ovarian cancer diagnosis: a population-based cohort study. BMC Cancer. 2013;13:85.

32. Assayag J, Pollak MN, Azoulay L. Post-diagnostic use of beta-blockers and the risk of death in patients with prostate cancer. Eur J Cancer. 2014;50:2838-2845.

33. Cata JP, Villarreal J, Keerty D, et al. Perioperative beta-blocker use and survival in lung cancer patients. J Clin Anesth. 2014;26:106-117.

34. Heitz F, du Bois A, Harter P, et al. Impact of beta blocker medication in patients with platinum sensitive recurrent ovarian cancer-a combined analysis of 2 prospective multicenter trials by the AGO Study Group, NCIC-CTG and EORTC-GCG. Gynecol Oncol. 2013;129: 463-466.

35. Heitz F, Hengsbach A, Harter P, et al. Intake of selective beta blockers has no impact on survival in patients with epithelial ovarian cancer. Gynecol Oncol. 2017;144:181-186.

36. Holmes S, Griffith EJ, Musto G, Minuk GY. Antihypertensive medications and survival in patients with cancer: a population-based retrospective cohort study. Cancer Epidemiol. 2013;37:881-885.

37. Jansen L, Weberpals J, Kuiper JG, et al. Pre- and post-diagnostic beta-blocker use and prognosis after colorectal cancer: results from a population-based study. Int J Cancer. 2017;141:62-71.

38. Livingstone E, Hollestein LM, van Herk-Sukel MP, et al. betaBlocker use and all-cause mortality of melanoma patients: results from a population-based Dutch cohort study. Eur J Cancer. 2013;49: 3863-3871.

39. Musselman RP, Li W, Gomes T, et al. Association between beta blocker usage and cancer survival in a large, matched population study among hypertensive patients. J Surg Res. 2014;186:639-640.

40. Parker WP, Lohse CM, Zaid HB, et al. Evaluation of beta-blockers and survival among hypertensive patients with renal cell carcinoma. Urol Oncol. 2017;35:36.e1-36.e6.

41. Sakellakis M, Kostaki A, Starakis I, Koutras A. Beta-blocker use and risk of recurrence in patients with early breast cancer. Chemotherapy. 2014;60:288-289. 
42. Shah SM, Carey IM, Owen CG, Harris T, Dewilde S, Cook DG. Does beta-adrenoceptor blocker therapy improve cancer survival? Findings from a population-based retrospective cohort study. Br J Clin Pharmacol. 2011;72:157-161.

43. Weberpals J, Jansen L, Haefeli WE, et al. Pre- and post-diagnostic beta-blocker use and lung cancer survival: a population-based cohort study. Sci Rep. 2017;7:2911.

44. Choi CH, Song TJ, Kim TH, et al. Meta-analysis of the effects of beta blocker on survival time in cancer patients. J Cancer Res Clin Oncol. 2014;140:1179-1188.

45. Weberpals J, Jansen L, Carr PR, Hoffmeister M, Brenner H. Beta blockers and cancer prognosis - the role of immortal time bias: a systematic review and meta-analysis. Cancer Treat Rev. 2016;47:1-11.

46. Zhong S, Yu D, Zhang X, et al. Beta-blocker use and mortality in cancer patients: systematic review and meta-analysis of observational studies. Eur J Cancer Prev. 2016;25:440-448.
47. Jean Wrobel L, Bod L, Lengagne R, Kato M, Prévost-Blondel A, Le Gal FA. Propranolol induces a favourable shift of anti-tumor immunity in a murine spontaneous model of melanoma. Oncotarget. 2016;7:77825-77837.

48. Partecke LI, Speerforck S, Kading A, et al. Chronic stress increases experimental pancreatic cancer growth, reduces survival and can be antagonised by beta-adrenergic receptor blockade. Pancreatology. 2016;16:423-433.

49. Ettinger DS, Wood DE, Aisner DL, et al. Non-small cell lung cancer, version 5.2017, NCCN clinical practice guidelines in oncology. J Natl Compr Canc Netw. 2017;15(4):504-535.

50. Barron AJ, Zaman N, Cole GD, Wensel R, Okonko DO, Francis DP. Systematic review of genuine versus spurious side-effects of betablockers in heart failure using placebo control: recommendations for patient information. Int J Cardiol. 2013;168(4):3572-3579. 


\section{Supplementary materials}

Table SI Quality assessment of the included studies

\begin{tabular}{|c|c|c|c|c|c|c|c|c|}
\hline \multirow[t]{4}{*}{ Subjects } & \multirow[t]{4}{*}{ Items } & \multirow[t]{4}{*}{ Standards } & \multicolumn{6}{|c|}{ Reference no. } \\
\hline & & & 8 & 9 & 10 & 11 & 12 & 13 \\
\hline & & & \multicolumn{6}{|l|}{ Score } \\
\hline & & & $\begin{array}{l}\text { Grytli } \\
\text { et al } \\
(2013)\end{array}$ & $\begin{array}{l}\text { Grytli } \\
\text { et al } \\
(2014)\end{array}$ & $\begin{array}{l}\text { Al-Niaimi } \\
\text { et al } \\
(2016)\end{array}$ & $\begin{array}{l}\text { Aydiner } \\
\text { et al } \\
(2013)\end{array}$ & $\begin{array}{l}\text { Barron } \\
\text { et al } \\
(201 \mathrm{I})\end{array}$ & $\begin{array}{l}\text { Beg } \\
\text { et al } \\
(20 \mid 7)\end{array}$ \\
\hline \multirow[t]{6}{*}{ Selection } & $\begin{array}{l}\text { I. Is the case } \\
\text { definition adequate? }\end{array}$ & $\begin{array}{l}\text { I. Yes, with independent } \\
\text { validation* } \\
\text { 2. Yes, eg, record linkage or } \\
\text { based on self-reports } \\
\text { 3. No description }\end{array}$ & 1 & 1 & 1 & 1 & 1 & 1 \\
\hline & $\begin{array}{l}\text { 2. Representativeness } \\
\text { of the cases }\end{array}$ & $\begin{array}{l}\text { I. Consecutive or obviously } \\
\text { representative series of } \\
\text { cases* } \\
\text { 2. Potential for selection } \\
\text { biases or not stated }\end{array}$ & 1 & 1 & 1 & 1 & 1 & 1 \\
\hline & 3. Selection of & I. Community controls* & 1 & & & & 1 & 1 \\
\hline & controls & $\begin{array}{l}\text { 2. Hospital controls } \\
\text { 3. No description }\end{array}$ & & 0 & 0 & 0 & & \\
\hline & $\begin{array}{l}\text { 4. Definition of } \\
\text { controls }\end{array}$ & $\begin{array}{l}\text { I. No history of disease (end } \\
\text { point)* }\end{array}$ & & & & & & \\
\hline & & 2. No description of source & 0 & 0 & 0 & 0 & 0 & 0 \\
\hline \multirow[t]{2}{*}{ Comparability } & $\begin{array}{l}\text { Comparability of } \\
\text { cases and controls on }\end{array}$ & $\begin{array}{l}\text { I. Study controls for the most } \\
\text { important factor* }\end{array}$ & 1 & 1 & 1 & 1 & 1 & 1 \\
\hline & $\begin{array}{l}\text { the basis of the design } \\
\text { or analysis }\end{array}$ & $\begin{array}{l}\text { 2. Study controls for any } \\
\text { additional factor (this } \\
\text { criteria could be modified } \\
\text { to indicate specific control } \\
\text { for a second important } \\
\text { factor*) }\end{array}$ & 1 & 1 & 1 & 1 & 1 & 1 \\
\hline \multirow[t]{4}{*}{ Exposure } & $\begin{array}{l}\text { I. Ascertainment of } \\
\text { exposure }\end{array}$ & $\begin{array}{l}\text { I. Secure record (eg, surgical } \\
\text { records)* } \\
\text { 2. Structured interview where } \\
\text { blind to case/control status* } \\
\text { 3. Interview not blinded to } \\
\text { case/control status } \\
\text { 4. Written self-report or } \\
\text { medical record only } \\
\text { 5. No description }\end{array}$ & 1 & 1 & 1 & 1 & 1 & 1 \\
\hline & $\begin{array}{l}\text { 2. Same method of } \\
\text { ascertainment for } \\
\text { cases and controls }\end{array}$ & $\begin{array}{l}\text { I. Yes* } \\
\text { 2. No }\end{array}$ & 1 & 1 & 1 & 1 & 1 & 1 \\
\hline & 3. Nonresponse rate & $\begin{array}{l}\text { I. Same rate for both groups* } \\
\text { 2. Nonrespondents described } \\
\text { 3. Rate different and no } \\
\text { designation }\end{array}$ & 1 & 1 & I & I & I & I \\
\hline & & & 8 & 7 & 7 & 7 & 8 & 8 \\
\hline
\end{tabular}




\begin{tabular}{|c|c|c|c|c|c|c|c|c|c|c|c|}
\hline 14 & 15 & 16 & 17 & 18 & 19 & 20 & 21 & 22 & 23 & 24 & 25 \\
\hline $\begin{array}{l}\text { Bir } \\
\text { et al } \\
(2015)\end{array}$ & $\begin{array}{l}\text { De Giorgi } \\
\text { et al } \\
(2013)\end{array}$ & $\begin{array}{l}\text { Diaz } \\
\text { et al } \\
(20 \mid 2)\end{array}$ & $\begin{array}{l}\text { Ganz } \\
\text { et al } \\
(2011)\end{array}$ & $\begin{array}{l}\text { Giampieri } \\
\text { et al } \\
(20 I 5)\end{array}$ & $\begin{array}{l}\text { Hwa } \\
\text { et al } \\
(20 \mid 7)\end{array}$ & $\begin{array}{l}\text { Jansen } \\
\text { et al } \\
(20 \mid 4)\end{array}$ & $\begin{array}{l}\text { Kim } \\
\text { et al } \\
(2017)\end{array}$ & $\begin{array}{l}\text { Lemeshow } \\
\text { et al } \\
(20 \mathrm{II})\end{array}$ & $\begin{array}{l}\text { Melhem- } \\
\text { Bertrandt } \\
\text { et al (20I I) }\end{array}$ & $\begin{array}{l}\text { Springate } \\
\text { et al } \\
(2015)\end{array}$ & $\begin{array}{l}\text { Udumyan } \\
\text { et al } \\
(20 \mid 7)\end{array}$ \\
\hline I & I & I & 1 & I & I & I & I & I & I & I & I \\
\hline
\end{tabular}

\begin{tabular}{|c|c|c|c|c|c|c|c|c|c|c|c|}
\hline I & I & I & I & I & I & I & 1 & I & I & I & I \\
\hline & I & & I & & & I & & I & & I & I \\
\hline 0 & & 0 & & 0 & 0 & & 0 & & 0 & 0 & \\
\hline 0 & 0 & 0 & 0 & 0 & 0 & 0 & 0 & 0 & 0 & 0 & 0 \\
\hline I & I & I & I & I & I & I & I & I & I & I & I \\
\hline I & 0 & I & I & I & I & I & 1 & I & I & 0 & I \\
\hline I & I & I & I & I & I & I & I & I & I & I & I \\
\hline I & I & I & I & I & I & I & I & I & I & I & I \\
\hline I & I & & & I & I & I & & & I & I & I \\
\hline & & 0 & 0 & & & & 0 & 0 & & & \\
\hline 7 & 7 & 6 & 7 & 7 & 7 & 8 & 6 & 7 & 7 & 7 & 8 \\
\hline
\end{tabular}


Table SI (Continued)

\begin{tabular}{|c|c|c|c|c|c|c|c|c|}
\hline \multirow[t]{4}{*}{ Subjects } & \multirow[t]{4}{*}{ Items } & \multirow[t]{4}{*}{ Standards } & \multicolumn{6}{|c|}{ Reference no. } \\
\hline & & & 26 & 27 & 28 & 29 & 30 & 31 \\
\hline & & & \multicolumn{6}{|l|}{ Score } \\
\hline & & & $\begin{array}{l}\text { Wang } \\
\text { et al } \\
(20 \mid 3)\end{array}$ & $\begin{array}{l}\text { Watkins } \\
\text { et al } \\
(20 \mid 5)\end{array}$ & $\begin{array}{l}\text { Yusuf } \\
\text { et al } \\
(20 \mid 2)\end{array}$ & $\begin{array}{l}\text { Botteri } \\
\text { et al } \\
(20 \mid 3)\end{array}$ & $\begin{array}{l}\text { Spera } \\
\text { et al } \\
(2017)\end{array}$ & $\begin{array}{l}\text { Johannesdottir } \\
\text { et al }(2013)\end{array}$ \\
\hline \multirow[t]{6}{*}{ Selection } & $\begin{array}{l}\text { I. Is the case } \\
\text { definition adequate? }\end{array}$ & $\begin{array}{l}\text { I. Yes, with independent } \\
\text { validation* } \\
\text { 2. Yes, eg, record linkage or } \\
\text { based on self-reports } \\
\text { 3. No description }\end{array}$ & 1 & 1 & $\mathrm{I}$ & I & I & 1 \\
\hline & $\begin{array}{l}\text { 2. Representativeness } \\
\text { of the cases }\end{array}$ & $\begin{array}{l}\text { I. Consecutive or obviously } \\
\text { representative series of } \\
\text { cases* } \\
\text { 2. Potential for selection } \\
\text { biases or not stated }\end{array}$ & 1 & 1 & I & 1 & 1 & 1 \\
\hline & 3. Selection of & I. Community controls* & & & & & & \\
\hline & controls & $\begin{array}{l}\text { 2. Hospital controls } \\
\text { 3. No description }\end{array}$ & 0 & 0 & 0 & 0 & 0 & 0 \\
\hline & $\begin{array}{l}\text { 4. Definition of } \\
\text { controls }\end{array}$ & $\begin{array}{l}\text { I. No history of disease (end } \\
\text { point)* }\end{array}$ & & & & & & \\
\hline & & 2. No description of source & 0 & 0 & 0 & 0 & 0 & 0 \\
\hline \multirow[t]{2}{*}{ Comparability } & $\begin{array}{l}\text { Comparability of } \\
\text { cases and controls on }\end{array}$ & $\begin{array}{l}\text { I. Study controls for the most } \\
\text { important factor* }\end{array}$ & 1 & 1 & 1 & 1 & I & 1 \\
\hline & $\begin{array}{l}\text { the basis of the design } \\
\text { or analysis }\end{array}$ & $\begin{array}{l}\text { 2. Study controls for any } \\
\text { additional factor (this } \\
\text { criteria could be modified } \\
\text { to indicate specific control } \\
\text { for a second important } \\
\text { factor*) }\end{array}$ & 1 & 0 & I & 1 & I & 1 \\
\hline \multirow[t]{5}{*}{ Exposure } & $\begin{array}{l}\text { I. Ascertainment of } \\
\text { exposure }\end{array}$ & $\begin{array}{l}\text { I. Secure record (eg, surgical } \\
\text { records)* } \\
\text { 2. Structured interview where } \\
\text { blind to case/control status* } \\
\text { 3. Interview not blinded to } \\
\text { case/control status } \\
\text { 4. Written self-report or } \\
\text { medical record only } \\
\text { 5. No description }\end{array}$ & 1 & I & I & I & I & 1 \\
\hline & $\begin{array}{l}\text { 2. Same method of } \\
\text { ascertainment for } \\
\text { cases and controls }\end{array}$ & $\begin{array}{l}\text { I. Yes* } \\
\text { 2. No }\end{array}$ & 1 & I & 1 & 1 & I & 1 \\
\hline & 3. Nonresponse rate & I. Same rate for both groups* & 1 & I & & I & I & \\
\hline & & $\begin{array}{l}\text { 2. Nonrespondents described } \\
\text { 3. Rate different and no } \\
\text { designation }\end{array}$ & & & 0 & & & 0 \\
\hline & & & 7 & 6 & 6 & 7 & 7 & 6 \\
\hline
\end{tabular}

Note: *Indicates I score. 
Dovepress

Beta-blocker use for cancer prognosis

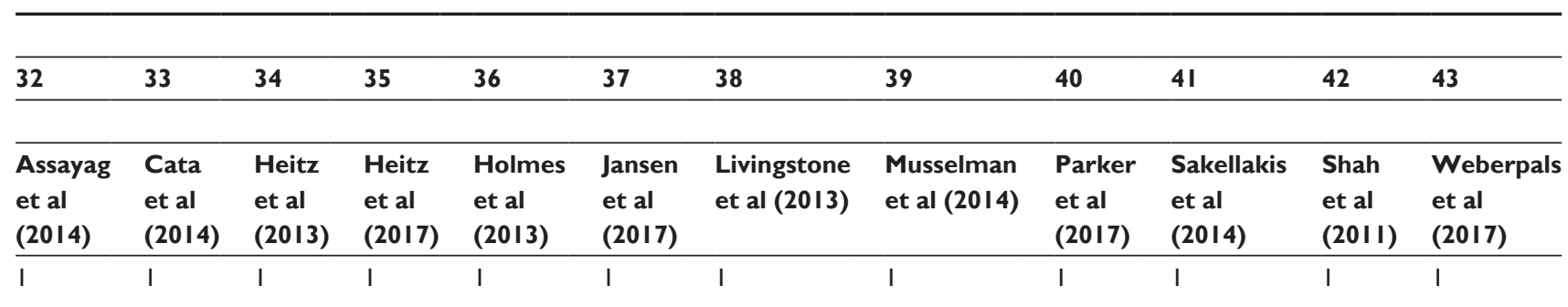

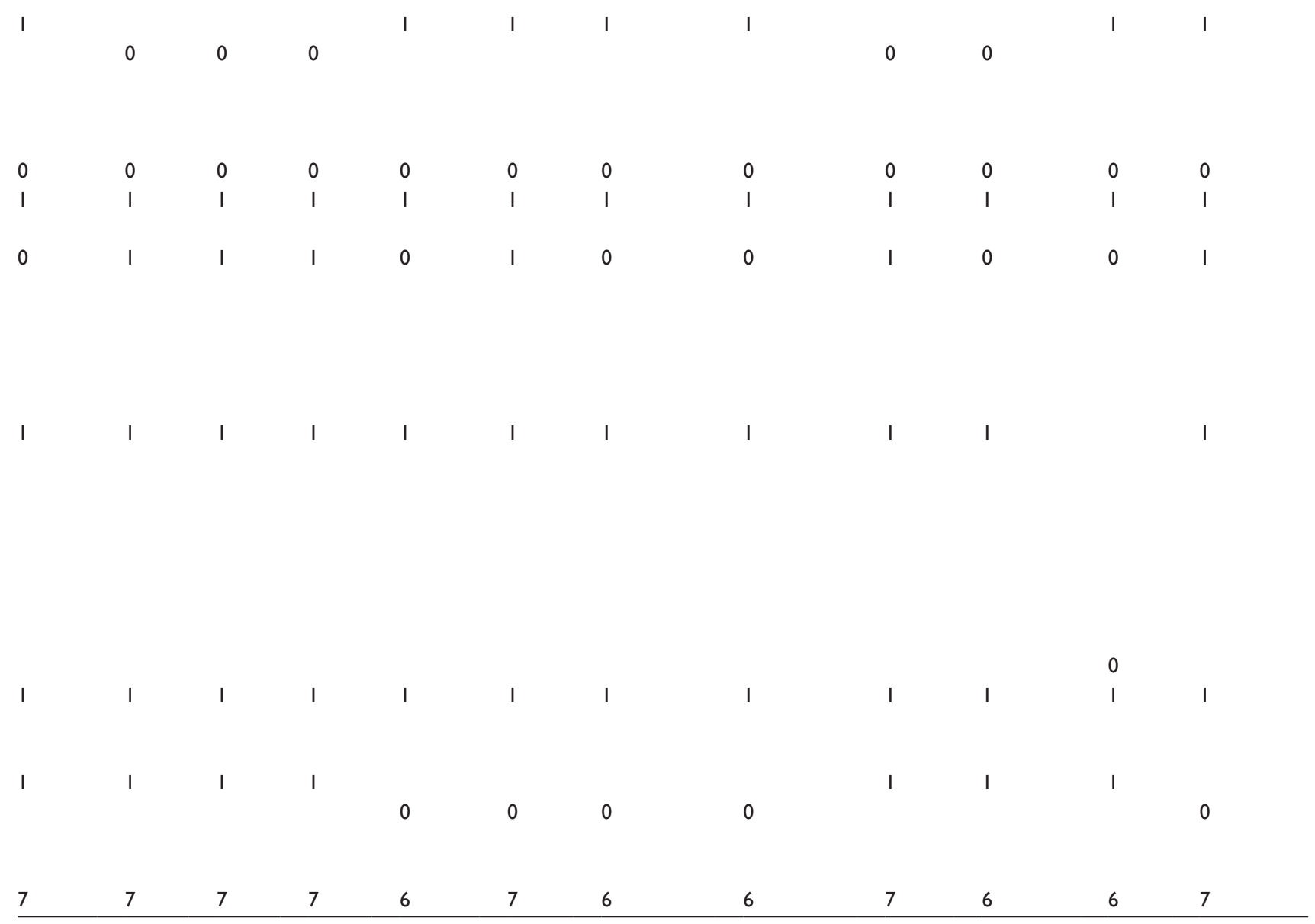

OncoTargets and Therapy 20I8: II

submit your manuscript | www.dovepress.com

Dovepress

4939 
A

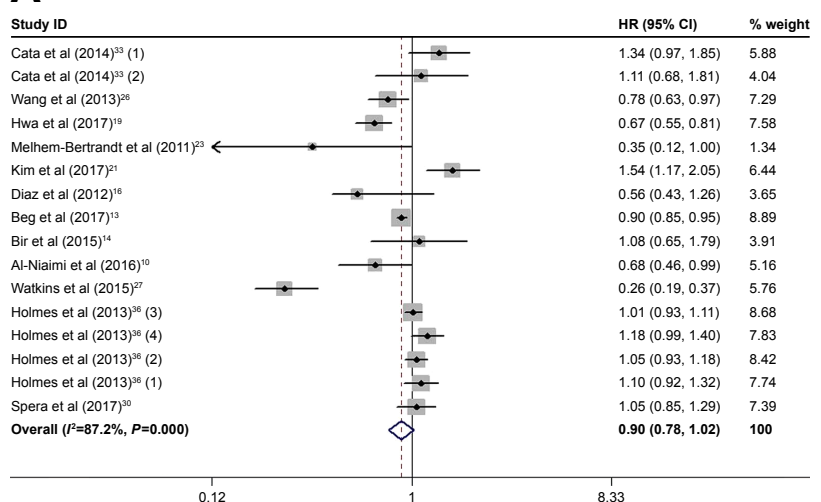

C

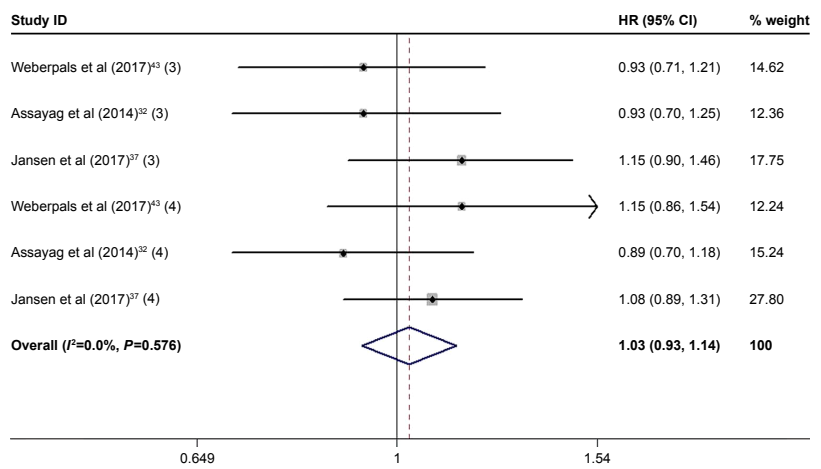

\section{E}

\section{Study ID}

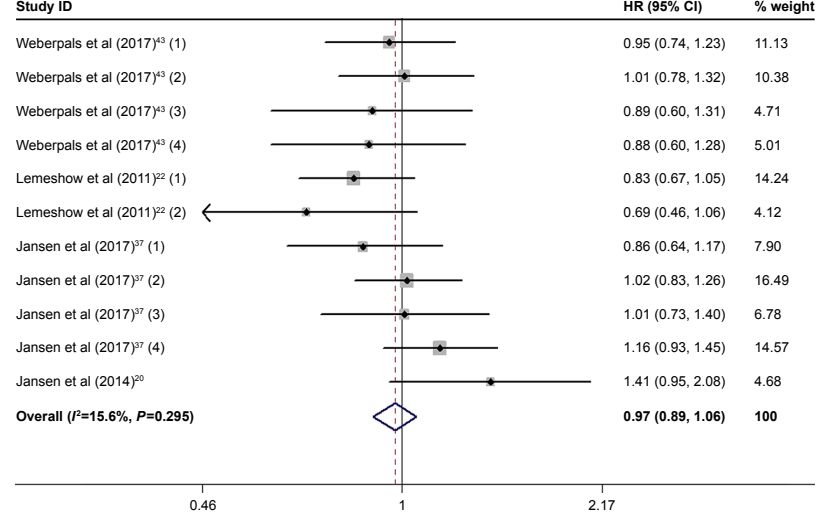

B

\begin{tabular}{|c|c|c|c|}
\hline Study ID & & HR $(95 \% \mathrm{Cl})$ & $\%$ weight \\
\hline Aydiner et al (2013)" & + & $0.69(0.36,1.34)$ & 2.84 \\
\hline Weberpals et al $(2017)^{43}(1)$ & - & $1.03(0.94,1.11)$ & 22.13 \\
\hline De Giorgi et al $(2013)^{15}$ & $\longleftarrow$ & $0.04(0.00,0.38)$ & 0.00 \\
\hline Shah et al $(2011)^{42}$ & - & $1.18(1.04,1.33)$ & 19.56 \\
\hline Udumyan et al $(2017)^{25}$ & - & $0.79(0.70,0.90)$ & 19.37 \\
\hline Heitz et al $(2017)^{95}$ & - & $0.94(0.69,1.29)$ & 9.01 \\
\hline Giampieri et al (2015) ${ }^{18}$ & + & $1.51(0.88,2.31)$ & 4.80 \\
\hline Weberpals et al $(2017)^{43}(2)$ & - & $1.00(0.92,1.08)$ & 22.30 \\
\hline Overall $(l=72.2 \%, P=0.001)$ & & $1.00(0.89,1.12)$ & 100 \\
\hline
\end{tabular}

D

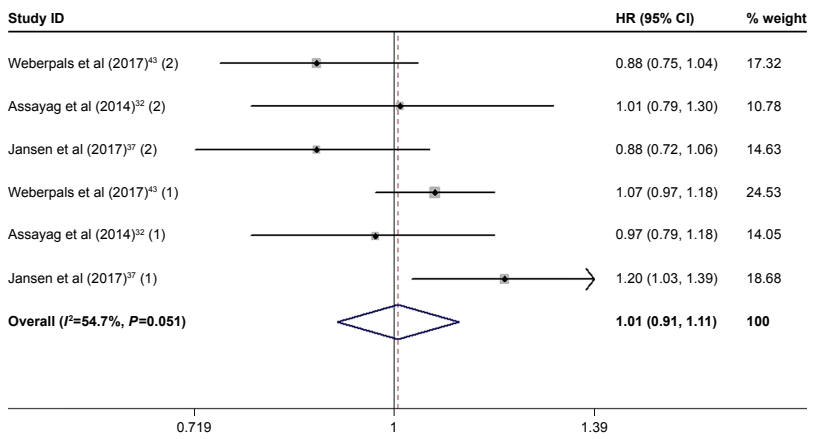

$\mathbf{F}$

Study ID \begin{tabular}{lllll}
\hline Weberpals et al $(2017)^{43}(1)$ & $\rightarrow$ & $0.87(0.75,1.02)$ & 11.35
\end{tabular} Weberpals et al $(2017)^{43}(2) \quad 0.94(0.80,1.10) \quad 11.12$ \begin{tabular}{l|lll} 
Weberpals et al $(2017)^{43}(3)$ & $1.20(1.06,1.35)$ & 12.68
\end{tabular} Weberpals et al $(2017)^{\mu 3}(4) \quad 1.17(1.03,1.33) \quad 12.40$ Lemeshow et al $(2011)^{22}(1) \quad 1.20(0.78,1.86) \quad 3.88$ Lemeshow et al $(2011)^{22}(2) \quad 0.93(0.48,1.80) \quad 1.95$ Jansen et al $(2017)^{37}(1) \quad 1.04(0.84,1.29) \quad 9.00$ Jansen et al $(2017)^{37}(2) \quad 1.16(0.95,1.43) \quad 9.36$ Jansen et al $(2017)^{37}(3) \quad 1.01(0.81,1.27) \quad 8.63$ Jansen et al $(2017)^{37}(4) \quad 1.11(0.90,1.36) \quad 9.29$ Jansen et al (2014) $)^{20}(1) \quad 1.01 .08(0.76,1.53) \quad 5.27$

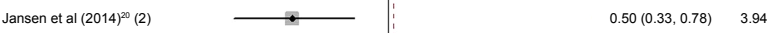
Watkins et al $(2015)^{27}$ Overall $\left(l^{2}=59.1 \%, P=0.003\right)$

Figure SI (Continued) 


\section{G}

Study ID Ganz et al (2011)
Beg et al $(2017)^{13}$

Beg et al (2017) $)^{13}$
Holmes et al (2013)

Weberpals et al (2017)

Barron et al (2011) 1$)^{12}(1)$

Holmes et al (2013) $)^{36}(1)$

Lemeshow et al (2011) ${ }^{22}$

Barron et al $(2011)^{12}(2)$

Udumyan et al (2017)

Shah et al $(2011)^{12}$

Holmes et al (2013)

Springate et al (2015) $)^{24}(1)$

Springate et al (2015) $)^{24}(2)$

Overall $(I=76.7 \%, P=0.000)$
Holmes et al (2013)

Weberpals et al $(2017)^{43}(2)$
$1.04(0.72,1.51) \quad 2.47$

$0.90(0.85,0.95) \quad 9.65$

$1.01(0.93,1.11) \quad 8.75$

$1.03(0.94,1.11) \quad 8.92$

$1.18(0.99,1.40) \quad 6.10$

$0.19(0.06,0.60) \quad 0.33$

$1.10(0.92,1.32) \quad 5.89$

$1.00(0.92,1.08) \quad 9.00$

$\begin{array}{ll}0.81(0.67,0.97) & 5.77 \\ 1.08(0.84,1.40) & 4.12\end{array}$

$0.79(0.70,0.90) \quad 7.57$

$1.18(1.04,1.33) \quad 7.66$

$1.05(0.93,1.18) \quad 7.78$

$1.03(0.93,1.14) \quad 8.34$

$1.18(1.04,1.33) \quad 7.66$

$1.01(0.94,1.08) \quad 100$
HR $(95 \% \mathrm{Cl}) \quad \%$ weight

H

\begin{tabular}{|c|c|c|c|}
\hline Study ID & & HR $(95 \% \mathrm{Cl})$ & $\%$ weight \\
\hline Cata et al (2014) 3$)^{33}(1)$ & - & $1.34(0.97,1.85)$ & 6.45 \\
\hline Cata et al $(2014)^{33}(2)$ & - & $1.11(0.68,1.81)$ & 5.36 \\
\hline De Giorgi et al $(2013)^{15}$ & & $0.04(0.00,0.38)$ & 0.00 \\
\hline Diaz et al $(2012)^{16}$ & - & $0.56(0.43,1.26)$ & 5.06 \\
\hline Heitz et al $(2017)^{35}$ & - & $0.94(0.69,1.29)$ & 6.52 \\
\hline Spera et al $(2017)^{30}$ & - & $1.05(0.85,1.29)$ & 7.10 \\
\hline Bir et al $(2015)^{14}$ & 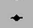 & $1.08(0.65,1.79)$ & 5.26 \\
\hline Yusuf et al (2012) & - & $0.64(0.51,0.81)$ & 6.98 \\
\hline Heitz et al $(2013)^{34}$ & - & $0.74(0.49,1.11)$ & 5.90 \\
\hline Al-Niaimi et al $(2016)^{10}$ & . & $0.68(0.46,0.99)$ & 6.07 \\
\hline Wang et al (2013) $)^{26}$ & - & $0.78(0.63,0.97)$ & 7.06 \\
\hline Aydiner et al (2013)"1 & - & $0.69(0.36,1.34)$ & 4.34 \\
\hline Kim et al $(2017)^{21}$ & & $1.54(1.17,2.05)$ & 6.71 \\
\hline Hwa et al $(2017)^{19}$ & & $0.67(0.55,0.81)$ & 7.17 \\
\hline Melhem-Bertrandt et al (2011 $)^{23}$ & & $0.35(0.12,1.00)$ & 2.56 \\
\hline Watkins et al $(2015)^{27}$ & & $0.26(0.19,0.37)$ & 6.39 \\
\hline Grytli et al (2013) & & $0.88(0.56,1.38)$ & 5.63 \\
\hline Giampieri et al (2015) & $\rightarrow$ & $1.51(0.88,2.31)$ & 5.42 \\
\hline Overall $\left(l^{2}=83.5 \%, P=0.000\right)$ & & $0.81(0.66,1.00)$ & 100 \\
\hline $1.0 \mathrm{e}$ & 1 & & \\
\hline
\end{tabular}

J

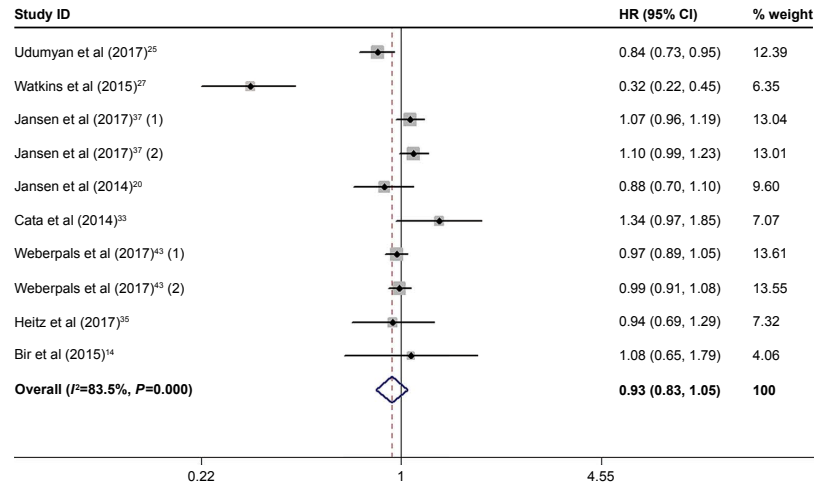

$\mathbf{L}$

Study ID

Weberpals et al (2017) Lemeshow et al (2011)22 Holmes et al (2013) ${ }^{36}(4)$ Udumyan et al (2017) $)^{25}$ Giampieri et al (2015) Shah et al $(2011)^{\text {s2 }}$ Holmes et al (2013) 3$)^{36}(3)$ Springate et al (2015) Springate et al (2015) Barron et al $(2011)^{12}(1)$ Barron et al (2011) 12 Holmes et al (2013) ${ }^{36}(1)$ Holmes et al (2013) ${ }^{36}(2)$ Overall $(P=74.7 \%, P=0.000)$ 0.03 HR $(95 \% \mathrm{Cl}) \quad \%$ weight $0.74(0.54,1.02) \quad 8.42$ $1.05(0.72,1.53) \quad 7.39$ $0.08(0.03,0.22) \quad 2.08$ $1.79(0.95,3.35) \quad 4.19$ $1.01(0.84,1.22) \quad 10.87$ $0.98(0.82,1.16) \quad 11.11$ $1.42(0.98,2.05) \quad 7.52$ $1.11(0.68,1.81) \quad 5.69$ $1.22(1.01,1.46) \quad 10.92$ $1.26(1.06,1.49) \quad 11.16$ $0.96(0.80,1.15) \quad 10.97$ $1.21(0.94,1.55) \quad 9.69$ $1.04(0.89,1.22) \quad 100$

33.3

HR $(95 \% \mathrm{Cl}) \quad \%$ weight

$1.00(0.92,1.08) \quad 10.80$ $0.81(0.67,0.97) \quad 7.37$

$1.18(0.99,1.40) \quad 7.74$

$0.79(0.70,0.90) \quad 9.34$

$1.51(0.88,2.31) \quad 2.25$

$1.18(1.04,1.33) \quad 9.43$

$1.01(0.93,1.11) \quad 10.55$

$1.03(0.93,1.14) \quad 10.13$

$1.18(1.04,1.33) \quad 9.43$

$0.19(0.06,0.60) \quad 0.47$

$1.08(0.84,1.40) \quad 5.44$

$1.10(0.92,1.32) \quad 7.51$

$1.05(0.93,1.18) \quad 9.56$

$1.03(0.95,1.11) \quad 100$

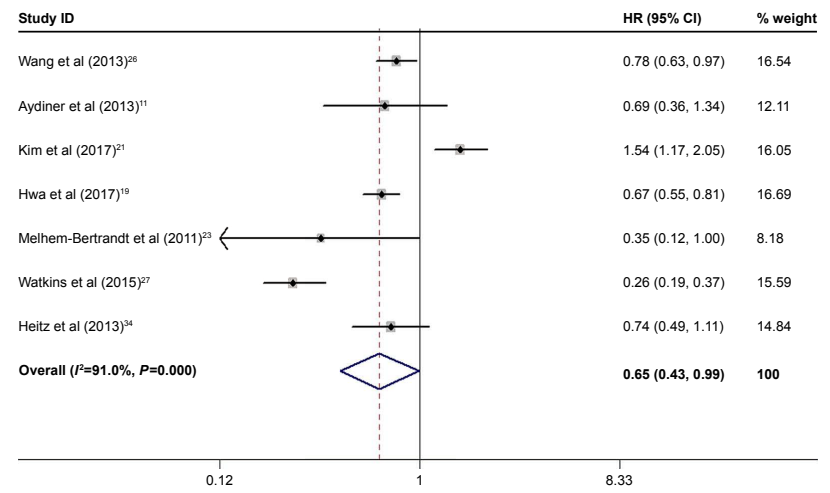

Figure SI (Continued) 


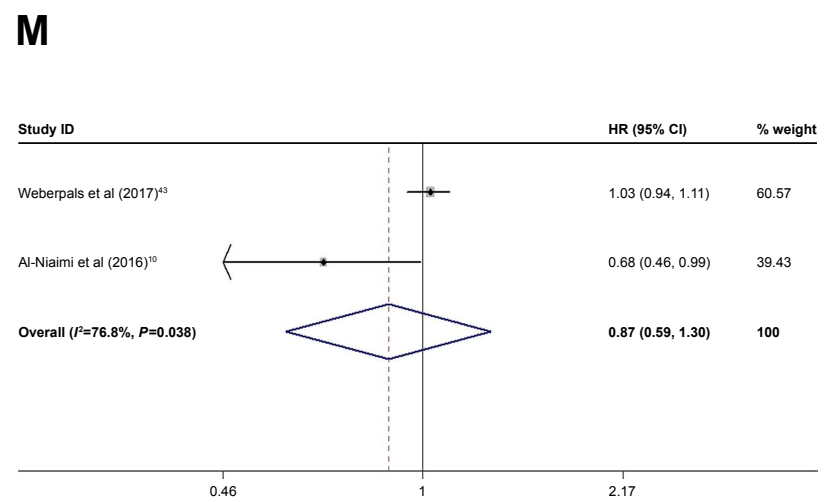

\section{$\mathbf{N}$}
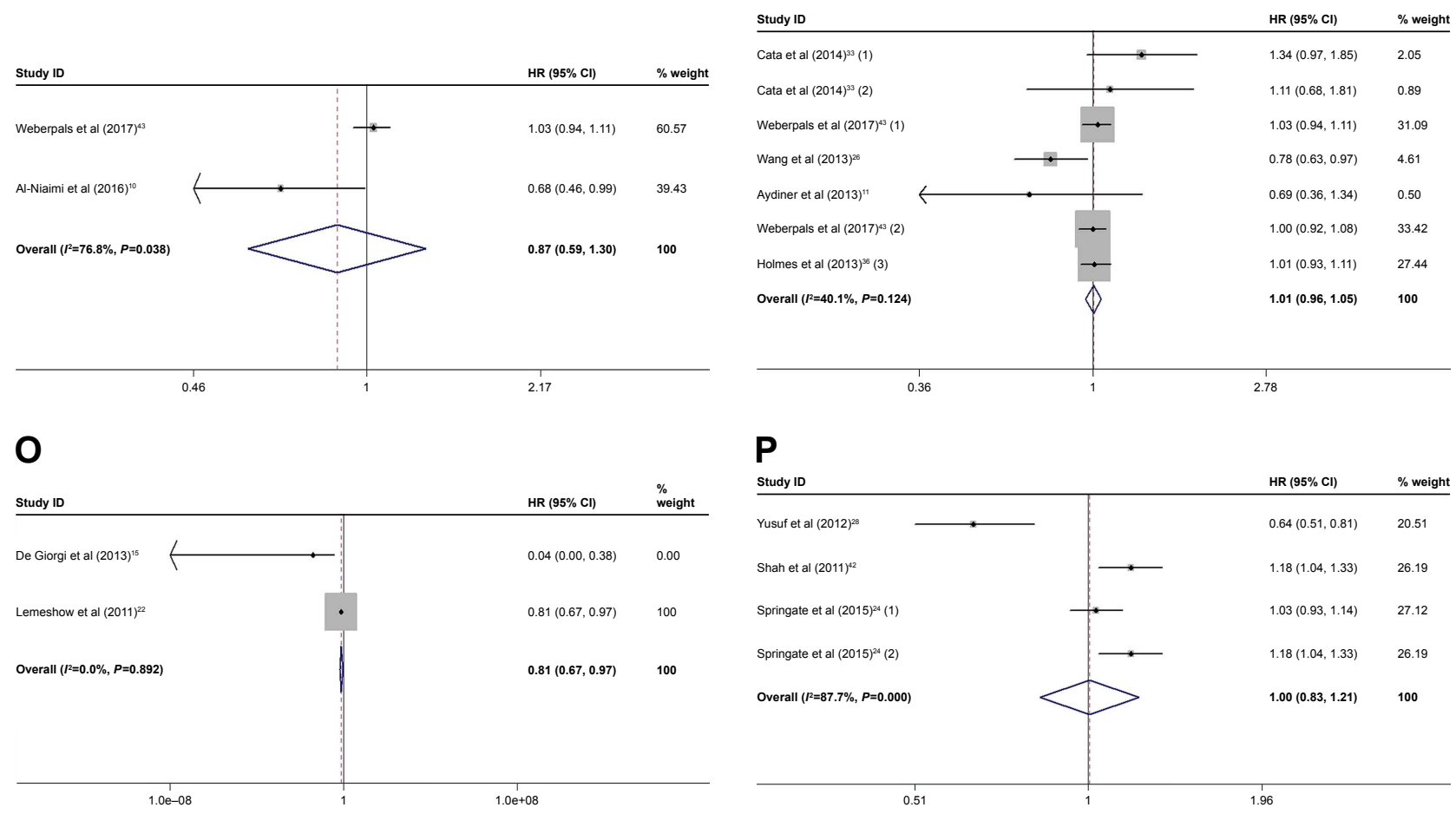

\section{$\mathbf{P}$}

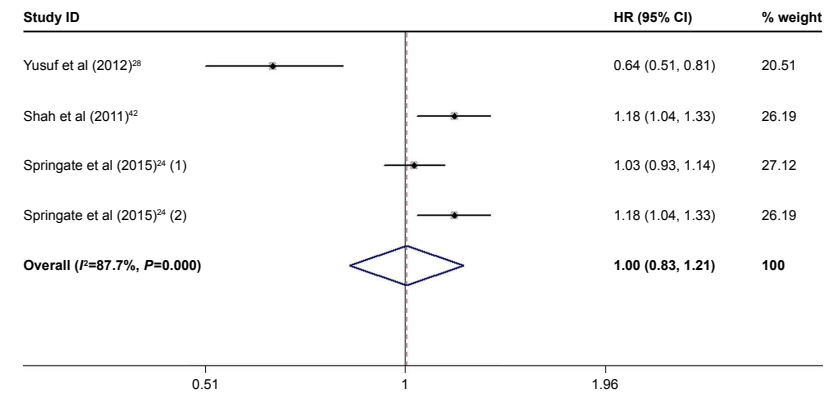

Q

\section{$\mathbf{R}$}

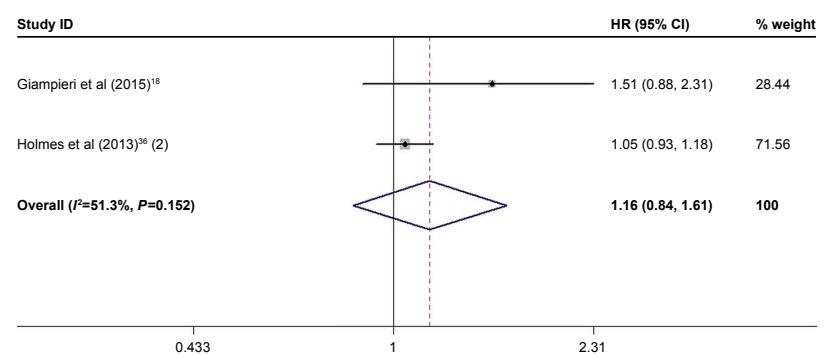

\section{Study ID}

HR $(95 \% \mathrm{Cl}) \quad \%$ weight

\begin{tabular}{ll|l|lll}
\hline Diaz et al (2012) $)^{16}$ & & & & $0.56(0.43,1.26)$ & 18.08
\end{tabular}

\begin{tabular}{l|llll} 
Heitz et al $(2017)^{35}$ & & & $0.94(0.69,1.29)$ & 21.05
\end{tabular}

\begin{tabular}{ll|rr} 
Watkins et al $(2015)^{27}$ & & $0.26(0.19,0.37)$ & 20.81
\end{tabular}

\begin{tabular}{lllll} 
Al-Niaimi et al $(2016)^{10}$ & \multicolumn{3}{c}{$0.68(0.46,0.99)$} & 20.20
\end{tabular}

$\begin{array}{lllll}\text { Heitz et al }(2013)^{34} & \longrightarrow & 0.74(0.49,1.11) & 19.86\end{array}$

Overall $(R=88.0 \%, P=0.000) \quad \longrightarrow \quad 0.59(0.36,0.96) \quad 100$

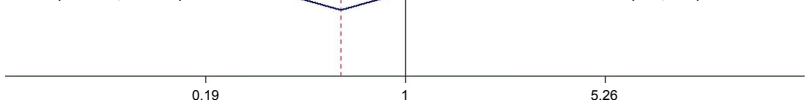

$\mathbf{T}$

S
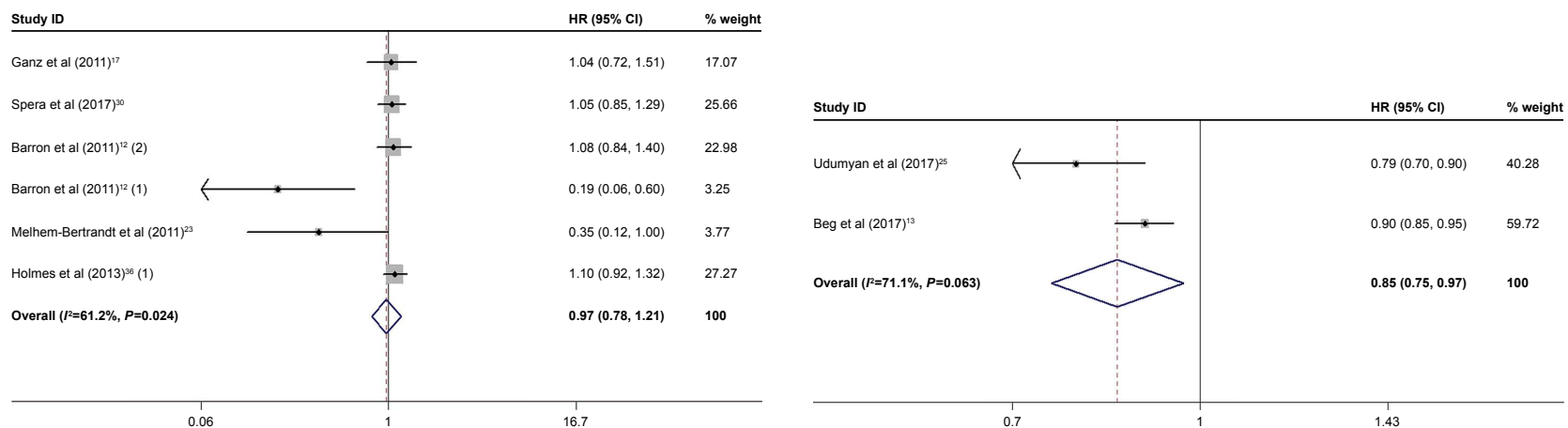

Figure SI Subgroup analysis on beta-blocker use and OS in patients with non-Europeans (A), Europeans (B); duration of drug use $>2$ years (C), duration of drug use $<2$ years (D); Stage I/II (E), Stage III/IV (F); sample size >80 (G), sample size $<80(\mathbf{H})$; non-selective beta-blocker (I), selective blocker-type (J); pre-diagnostic beta-blocker use (K), post-diagnostic beta-blocker use (time-fixed) (L), post-diagnostic beta-blocker use (time-dependent) (M); lung cancer $(\mathbf{N})$, melanoma $(\mathbf{O})$, mixed cancer $(\mathbf{P})$, colorectal cancer $(\mathbf{Q})$, ovarian cancer $(\mathbf{R})$, breast cancer $(\mathbf{S})$, and pancreatic cancer $(\mathbf{T})$.

Note: Weights are from random-effects analysis. The numbers in parentheses indicate the different included studies in the same year. 


\section{A}

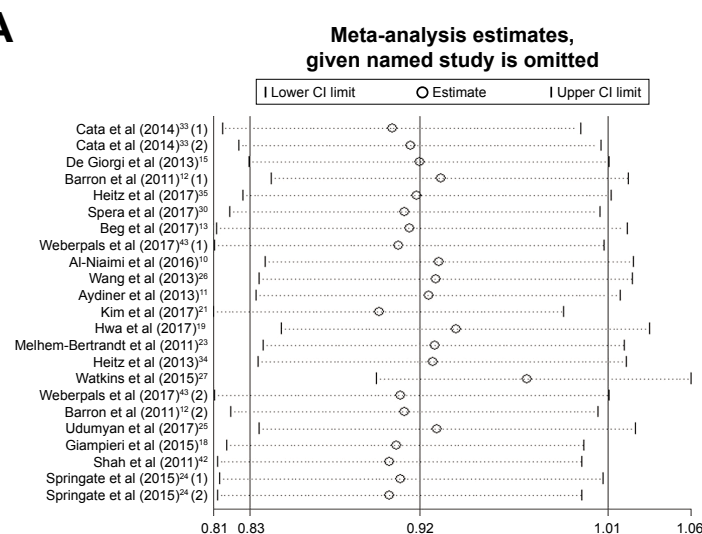

C
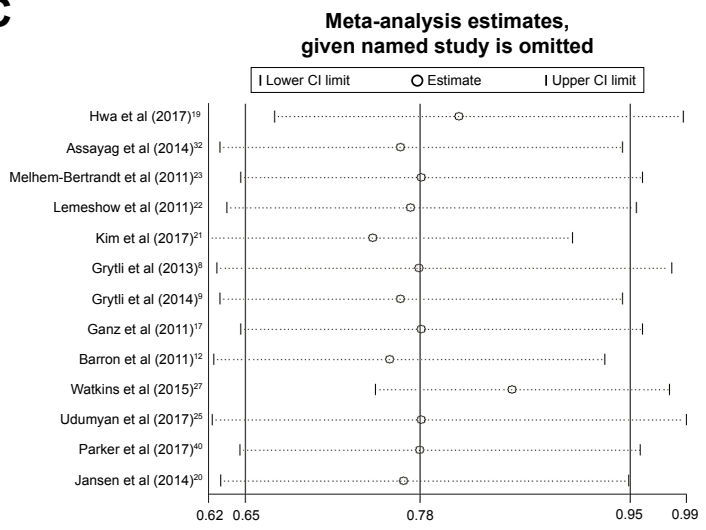

E

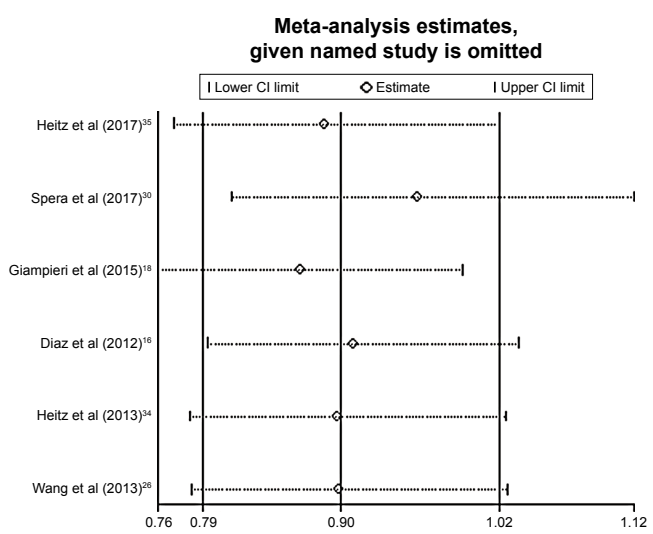

B

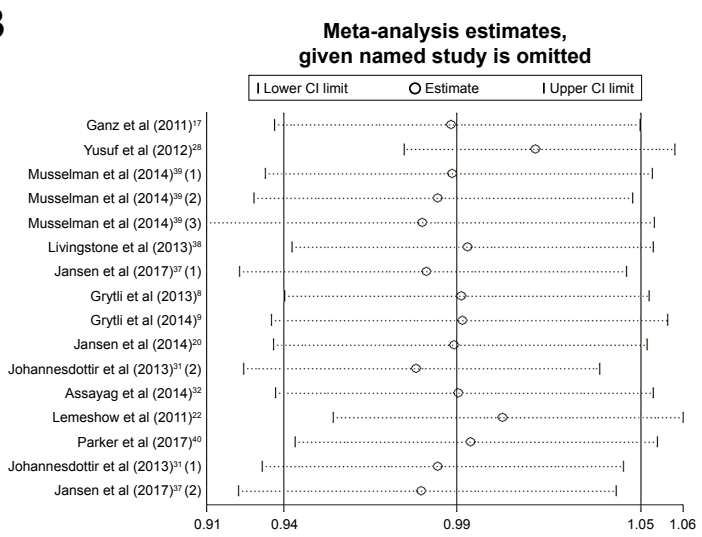

D

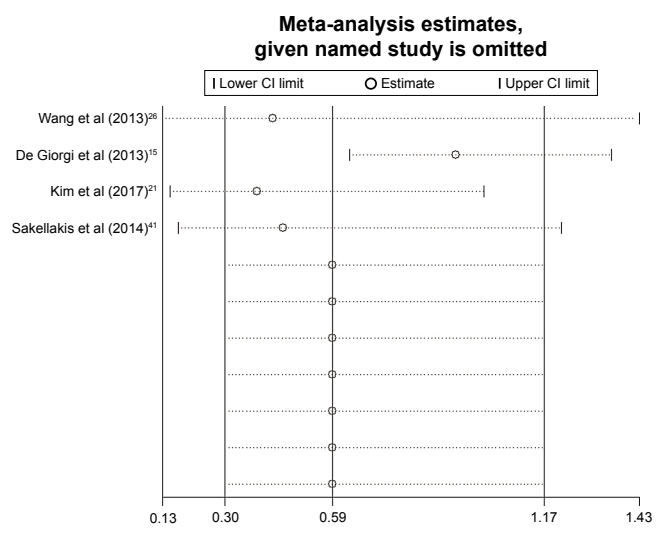

$\mathbf{F}$

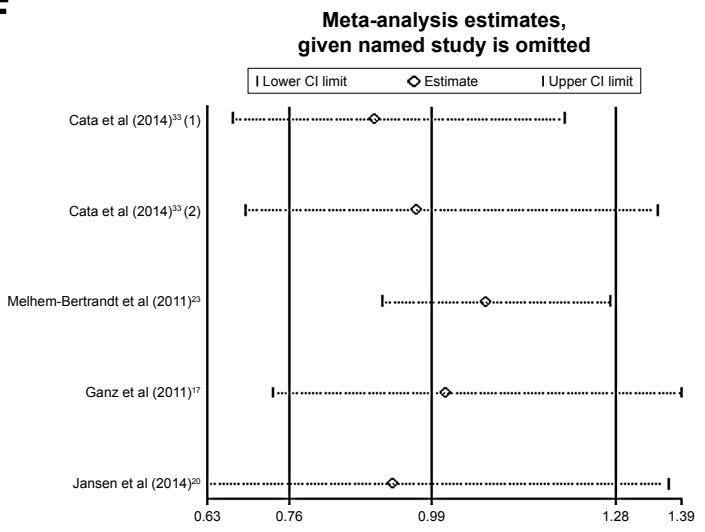

Figure S2 Sensitivity analysis of beta-blocker use on OS (A), all-cause mortality (B), CSS (C), DFS (D), PFS (E), and RFS (F) in studies except the studies obtaining estimates from KM plots.

Abbreviations: OS, overall survival; CSS, cancer-specific survival; DFS, disease-free survival; PFS, progression-free survival; RFS, recurrence-free survival; KM, kaplan-meier. 
A

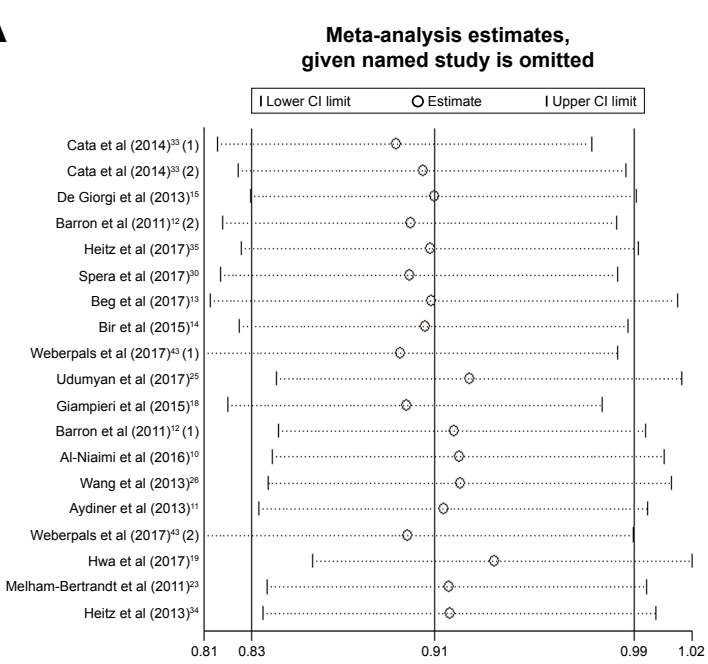

C

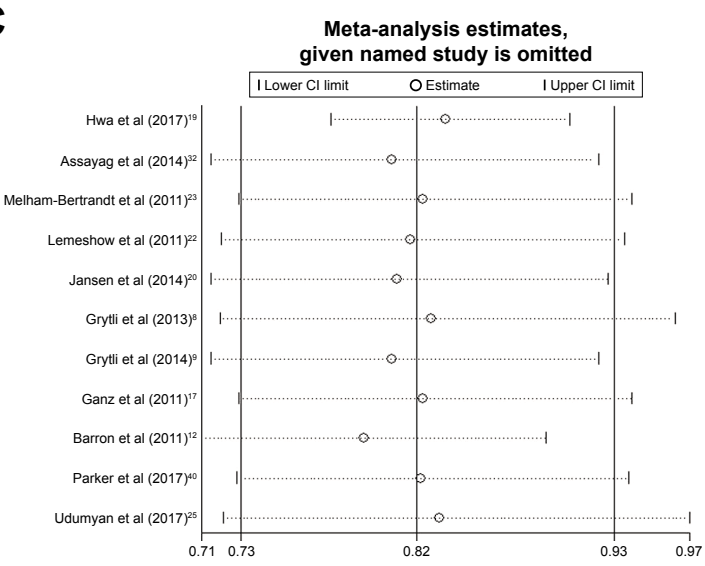

B

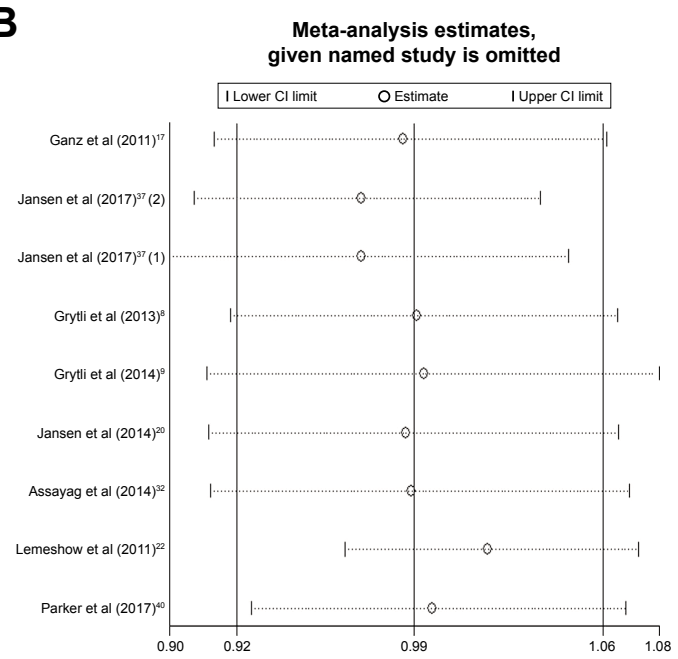

D

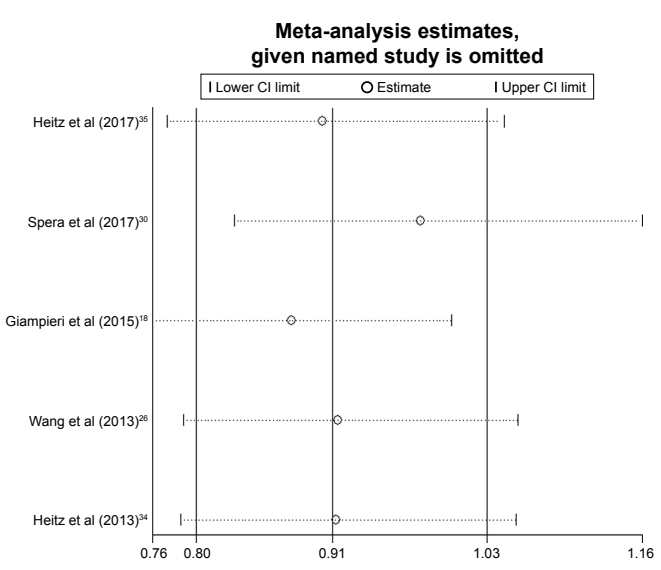

$\mathbf{E}$

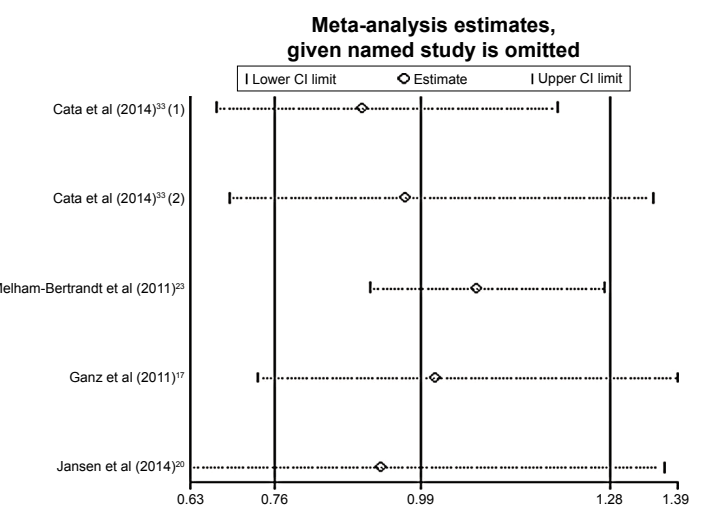

Figure S3 Sensitivity analysis of beta-blocker use on OS (A), all-cause mortality (B), CSS (C), PFS (D), and RFS (E) in high-quality studies (NOS score $\geq 7$ ). Abbreviations: OS, overall survival; CSS, cancer-specific survival; PFS, progression-free survival; RFS, recurrence-free survival; NOS, Newcastle-Ottawa Quality Assessment Scale.

\section{Publish your work in this journal}

OncoTargets and Therapy is an international, peer-reviewed, open access journal focusing on the pathological basis of all cancers, potential targets for therapy and treatment protocols employed to improve the management of cancer patients. The journal also focuses on the impact of management programs and new therapeutic agents and protocols on patient perspectives such as quality of life, adherence and satisfaction. The manuscript management system is completely online and includes a very quick and fair peer-review system, which is all easy to use. Visit http://www.dovepress.com/testimonials.php to read real quotes from published authors. 